\title{
RÍO BOTE 1 (SANTA CRUZ, ARGENTINA): ANÁLISIS TAFONÓMICO DE LOS CONJUNTOS ZOOARQUEOLÓGICOS RECUPERADOS ENTRE FINES DEL HOLOCENO MEDIO Y TARDÍO
}

\author{
MARÍA VICTORIA FIEL ${ }^{a} \&$ LAURA MARCHIONNI ${ }^{\mathrm{b}}$
}

\begin{abstract}
RESUMEN
En este trabajo realizamos un estudio exploratorio de los procesos de formación del sitio RB1, a partir del análisis del registro zooarqueológico comprendido en capas estratificadas entre ca. 4.200 y 350 años AP. Para ello evaluamos el grado de intervención de diferentes agentes y estudiamos la preservación e integridad del registro en relación con los cambios en la morfología del sitio para discutir el rol humano en la formación de los conjuntos. Los resultados indican que el ser humano habría sido el principal agente acumulador y que su accionar habría variado a lo largo de la secuencia analizada. A su vez, el registro faunístico sugiere cambios en la preservación de los restos óseos, los cuales se encuentran en estrecha relación con las variaciones ambientales del área.
\end{abstract}

PALABRAS CLAVE: procesos de formación de sitio, Patagonia meridional, Holoceno temprano, Holoceno tardío.

RÍO BOTE 1 (SANTA CRUZ, ARGENTINA):

TAPHONOMIC ANALYSIS OF THE ZOOARCHAEOLOGICAL ASSEMBLAGES RECOVERED BETWEEN LATE MIDDLE HOLOCENE AND LATE HOLOCENE

\begin{abstract}
In this paper we perform an exploratory study of the formation processes involved in the case of RB1 site, based on the analysis of the zooarchaeological record of stratified layers dated between ca. 4.200 and 350 years BP. To do that, we evaluated the degree of intervention of different agents and studied the preservation and the integrity of the zooarchaeological record in relation to changes in site morphology, to be able to discuss the human role in the formation of assemblages. The results indicate that humans were the main accumulator agent, and their actions have varied throughout the analyzed sequence. The zooarchaeological record suggests changes in the preservation of bone remains, which, according to the results are closely related to environmental variations in the area.

a Departamento de Ciencias Antropológicas, Facultad de Filosofía y Letras, Universidad de Buenos Aires.

mvictoriafiel@gmail.com

b CONICET, División de arqueología, Facultad de Ciencias Naturales y Museo - UNLP. lau_marchionni@yahoo.com.ar
\end{abstract}


KEY WORDS: site formation processes, Southern Patagonia, Middle Holocene, Late Holocene.

\section{INTRODUCCIÓN}

Los trabajos arqueológicos realizados al sur de la cuenca superior del río Santa Cruz, sugieren una lenta incorporación del área dentro del rango de acción de los grupos cazadores-recolectores que habitaron la región desde ca. 9.700 años AP (Franco et al. 1999, 2016, 2018; Franco, 2002). Las investigaciones sobre el poblamiento humano de este espacio se han centralizado en la circulación de los grupos humanos y en las diferentes etapas de en las que se llevó a cabo la ocupación del área, siguiendo el modelo de poblamiento planteado por Borrero (199495) para Patagonia (Franco, 2002; Franco et al. 2004). Un problema que ha tenido especial relevancia en la discusión de dichas etapas, ha sido el abandono o uso discontinuo de ciertas zonas y el reposicionamiento de los grupos humanos en función de los cambios climáticos registrados durante el Holoceno medio-tardío (Borrero \& Franco, 2000; Franco et al. 2004; Franco et al. 2018). Para dichos momentos se ha registrado una relación inversa de las precipitaciones entre la zona ubicada al este del área y la zona cercana a los Andes (Mancini, 2002; Tonello et al. 2009 en Franco et al. 2018; Mancini et al. 2013; Ohlendorf et al. 2014; Strelin et al. 2014). En consecuencia, se ha sugerido que el cambio ambiental influyó en la movilidad y los tipos de asentamientos al oeste y al este del Lago Argentino (Franco et al. 2016, 2018).

En este marco, investigaciones como las desarrolladas por Otaola $(2008,2009)$ y Otaola \& Franco (2008) en el sitio Chorrilo Malo 2 (Fig. 1) han dado cuenta de cómo la variabilidad climática local habría influido en el aprovechamiento humano de los recursos animales. Estas investigaciones, a su vez, plantearon la idea de que las discontinuidades en el uso de este espacio por parte de los grupos cazadores-recolectores estarían en relación tanto con las variaciones ambientales como con las distintas etapas de poblamiento del área (Otaola, 2008, 2009). Dichas investigaciones pusieron en juego variables tafonómicas, como la evidencia de marcas de carnívoros y huellas antrópicas sobre los restos óseos, para dar cuenta de la continuidad del uso humano del sitio y generar expectativas faunísticas sobre el modelo de poblamiento del área. Sin embargo, en otros sitios del área no se han llevado a cabo aún investigaciones arqueofaunísticas como las mencionadas para el caso de Chorrillo Malo 2. De esta manera, y en el contexto de las investigaciones acerca de cómo se llevó adelante el poblamiento de este sector de Patagonia y su relación con los cambios ambientales, se vuelve necesario abordar el estudio zooarqueológico y tafonómico de estos sitios a fin de aportar nueva información para el área, que contribuya a la comprensión de los procesos y dinámicas poblacionales y su relación con los sectores aledaños.

El sitio Río Bote 1 (RB1) es un abrigo rocoso de arenisca, localizado en la margen derecha del curso inferior del río homónimo, a 5 $\mathrm{km}$ de distancia en línea recta del río Santa Cruz y a $75 \mathrm{~km}$ hacia el este del sitio Chorrillo Malo 2 (Fig. 1; Mehl \& Franco, 2009). La secuencia sedimentaria arqueológica está conformada por capas de origen cultural con fogones, y abundante material lítico, faunístico y óseo humano, fechadas entre el Holoceno medio y tardío (Franco et al. 2010, 2016, 2017a y b, 2018; Guarido, 2014; Vetrisano, 2017; Fiel, 2019). A lo largo de su ocupación se registran cambios culturales y variaciones ambientales (Franco et al. 2016, 2018), que han permitido incorporar la zona del valle de río Bote a las discusiones acerca de la influencia del ambiente sobre las ocupaciones del área (Franco, 2002; Franco \& Borrero, 2003; Otaola, 2008; Franco et al. 2016, 2017a, 2018).

En este trabajo, nuestro objetivo principal consiste en estudiar, a partir del registro zooarqueológico, los procesos de formación del sitio RB1 y discutir el rol humano en la formación de los conjuntos. Para ello realizamos un análisis tafonómico de la fauna fechada entre ca. 4.200 y 350 años AP, evaluamos el grado de intervención de diferentes agentes (carnivoros, roedores y humanos) y, a partir de esta información, estudiamos la preservación y la integridad del registro. Luego analizamos la relación de las variaciones ambientales registradas en el área de 


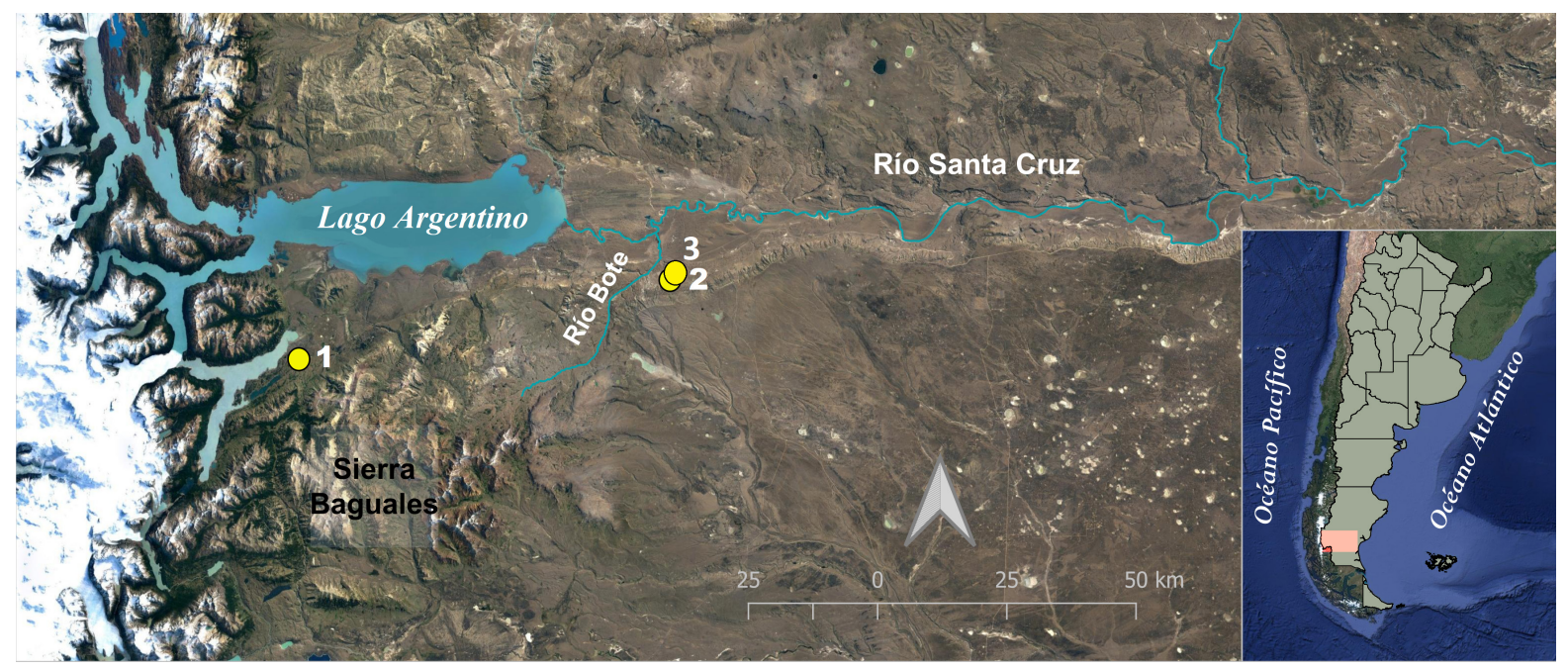

Fig.1. Sur de la cuenca superior del río Santa Cruz.

Referencias: 1: Chorrillo Malo 2; 2: Río Bote 1; 3: Río Bote 2.

RB1 y los cambios en la morfología del sitio para comprender la presencia humana en el alero. Finalmente, para lograr una visión de mayor alcance sobre el uso humano del espacio en el área, discutimos los resultados aquí alcanzados con los antecedentes de Chorrillo Malo 2.

\section{RÍO BOTE 1}

El sitio comenzó a ser trabajado en el año 2007 cuando pobladores locales observaron la presencia de restos humanos en el perfil del alero y al pie del sitio, luego de que un cambio en el curso del río provocara la exposición de su secuencia sedimentaria (Mehl \& Franco, 2009). Las crecidas anuales del río amenazaban la integridad de RB1 $\mathrm{y}$ ocasionaban que éste se encontrara en riesgo de derrumbe, aspecto que motivó la recuperación de los restos humanos (Franco et al. 2010, 2017a). Desde el momento del hallazgo del sitio hasta la actualidad se realizaron una gran variedad de estudios sobre distintas materialidades (e.g. Franco, 2008, 2009; Mehl \& Franco, 2009; Franco et al. 2010, 2011, 2016, 2017a, 2018; Guarido, 2014; Franco \& Vetrisano, 2014; Fiel, 2019, 2020).

\section{Descripción geomorfológica y cronológica del sitio}

Río Bote 1 se encuentra localizado en una cota de ca. 215 metros de altura (Mehl \& Franco,
2009), sobre la margen este del curso inferior del río homónimo cuya cabecera se encuentra en la sierra Baguales y es el primer afluente de la margen derecha del río Santa Cruz (Fig. 1). La erosión del sitio se considera principalmente hídrica -por los deshielos estacionales que producen derrumbes en el alero-; pero también es eólica, debido a que el alero está orientado hacia el oeste, de donde provienen los vientos predominantes (Mehl \& Franco, 2009). El corte de la secuencia estratigráfica y la presencia de grandes bloques de arenisca sumergidos en el río en las inmediaciones del sitio, sugiere que en el pasado el abrigo rocoso habría sido más grande, extendiéndose hacia el oeste (Mehl \& Franco, 2009).

Se trata de un abrigo rocoso de arenisca con Ostreas, con una su secuencia sedimentaria que mide aproximadamente $2,50 \mathrm{~m}$ de espesor y con un ancho de aproximadamente 1 metro desde la pared del alero hasta el río (Mehl \& Franco, 2009). La sucesión de depósitos está compuesta por sedimentos eólicos, hídricos y otros que son producto del desprendimiento de la roca del alero (Mehl \& Franco, 2009).

El techo del alero posee una grieta por la cual se filtra agua, lo que habría favorecido el crecimiento de vegetación sobre parte de la superficie de la columna sedimentaria excavada (Mancini com. pers. en Guarido, 2014; Franco et al. 2017a). Durante los trabajos de campo se 
registró la presencia de un nido actual de un ave pequeña, dentro de uno de los cráneos humanos recuperados y un pozo de roedor en la mitad superior de la secuencia sedimentaria, próximo a la pared del afloramiento (Guarido, 2014; Franco et al. 2017a).

Los fechados sobre restos óseos y carbones recuperados del perfil del sitio dieron cuenta de una serie de fechados consistentes comprendidos entre ca. 5.800 y 350 años AP (ca. 6.630-6.410 y 450 años cal. AP) (Fig. 2). Por otro lado, los fechados realizados sobre las capas excavadas indicaron una secuencia sedimentaria comprendida entre al menos ca. 4.200 años AP (4.820-4.570 años cal. AP) y ca. 3.600 años AP (4.086-3.840 años cal. $\mathrm{AP})$. Además, se registró una capa de tefra procede del volcán Aguilera (Stern com. pers. en Franco, 2009) la cual posee fechados comprendidos entre ca. 4.030 y 3.900 años AP (4.560-4.090 años cal. AP; Franco et al. 2017a, 2018). Tanto la capa de tefra, como las capas fechadas entre ca. 4.200 y 3.900 años AP se encuentran cortadas por las estructuras de entierro en forma de pozo en el extremo sur del alero (Fig. 2).

Se documentaron en el sitio al menos cuatro estructuras de entierro. Las primeras tres se encontraban superpuestas y fueron fechadas entre ca. 3.800 y 3.600 años AP (ca. 4.200 a 3.700 años cal. AP), momento a partir del cual se registra un hiato en los fechados hasta ca. 2.100 años AP (ca. 2.300-2.000 años cal. AP), este corresponde al cuarto pozo de entierro. Esta última estructura de entierro estaba ubicada inmediatamente encima de las estructuras previas (Franco et al. 2010, 2011, 2017a). Luego de dicho fechado se registró un segundo hiato en los fechados, que se prolonga hasta ca. 350 años AP (ca. 450 años cal. AP), momento en el cual se registra un pequeño fogón con escasos restos materiales (Franco et al. 2010, 2011, 2017a, 2018).

\section{Investigaciones arqueológicas}

Las evidencias de ocupación humana en este sitio datan de inicios del Holoceno medio, prolongándose de manera discontinua hasta hace ca. 350 años AP (Mehl \& Franco, 2009; Franco et al. 2017a, 2018). La ocupación más temprana detectada data de ca. 5.800 años AP -donde se recuperó una concentración de carbones (estructura de combustión) junto con escasos restos faunísticos y artefactos líticos (Franco et al. 2018)-,

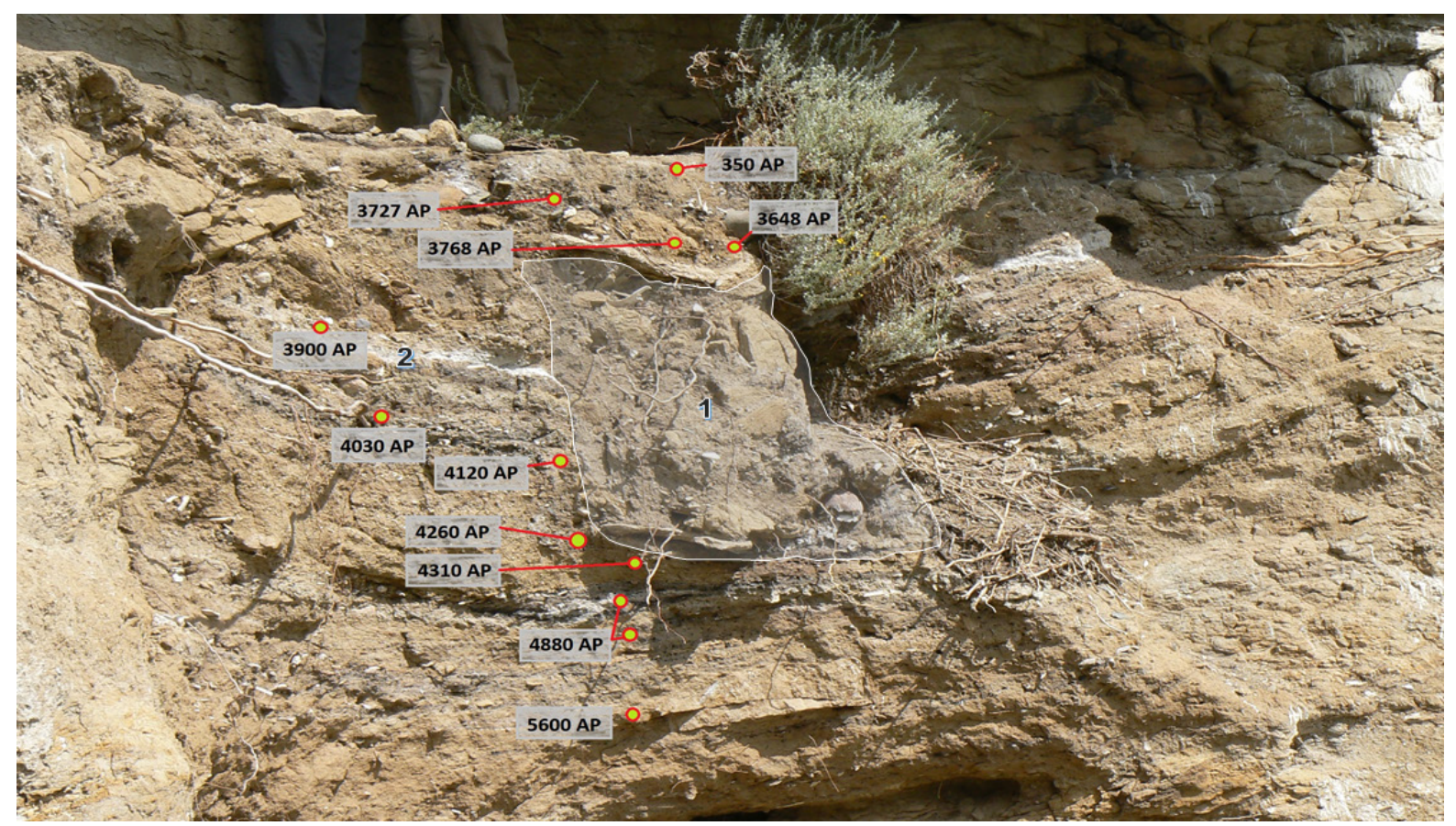

Fig. 2. Secuencia estratigráfica de Río Bote 1 con fechados. Referencias: 1: entierros humanos; 2: cenizas del volcán Aguilera. 
esta ocupación se corresponde con un periodo de descenso de la humedad en la estepa (Franco et al. 2016). Sin embargo, las evidencias antrópicas solo comienzan a ser abundantes a partir de ca. 4.200 años AP, sugiriendo un uso reiterado del sitio a partir de ese momento (Franco et al. 2018).

La información paleoambiental de la zona del valle de río Bote indica que, dentro de un contexto general de aridez, la humedad decrece hasta ca. 4.300 años AP (ca. 4.800-4.600 años cal. AP), luego incrementa levemente hacia ca. 4.260 años $\mathrm{AP}$ (ca. 4.800-4.600 años cal. AP) y nuevamente disminuye hacia ca. 4.100 años AP (ca. 4.400 años cal. AP; Franco et al. 2016; 2018).

Los trabajos sobre similitudes tecnológicas del área han señalado que, entre ca. 4.300-3.800 y 3.600 años AP, RB1 estaba dentro del rango de acción de grupos culturales locales, utilizando los espacios ubicados al sur y al oeste del Lago Argentino, probablemente también la sierra Baguales (Franco et al. 2011, 2018; Franco \& Vetrisano, 2014). Hacia ca. 3.900 años AP se registra en $\mathrm{RB} 1$ el uso de tecnología ósea con la presencia de una punta circular realizada sobre un hueso largo de taxón indeterminado (Franco et al. 2017a). Por otro lado, hacia ca. 3.900 años $\mathrm{AP}$ se documenta en el sitio un reemplazo de la tecnología laminar por el denominado método de talla Levallois recurrente centrípeto, el cual está presente en el área desde ca. 4.300 ó 3.800 años AP (Franco \& Vetrisano, 2014; Franco et al. 2018). Este método continuó siendo utilizando, en RB1, al menos hasta ca. 3.600 años AP (Franco et al. 2017a, 2018).

Las tres primeras estructuras de entierro pertenecen a un periodo en el cual se registran condiciones extremadamente áridas en la estepa, al este del lago Argentino, pero más húmedas que las identificadas hacia el oeste del área (Franco et al. 2018). Inmediatamente por encima de las rocas que tapan los entierros hay depósitos culturales con evidencia de fogones, materiales líticos $y$ restos faunísticos. Los fechados correspondientes a estos depósitos se encuentran en el mismo rango cronológico que los cuerpos (ca. 3.800-3.600 años $\mathrm{AP}$ ), lo que sugiere que las ocupaciones humanas de dichos momentos probablemente fueron penecontemporáneas con uno o más de los eventos de entierro (Franco et al. 2010, 2017a).
La secuencia estratigráfica continúa con el entierro aislado fechado en ca. 2.100 años $\mathrm{AP}$, ubicado sobre las estructuras de entierro previas. La evidencia paleoclimática disponible sugiere condiciones levemente más húmedas en el valle del río Bote, donde se encuentra el sitio -en comparación a momentos previos-, por el contrario, hacia el oeste, se registra el comienzo de condiciones más bien áridas y fluctuantes (Franco et al, 2018) (Tabla 1). La evidencia de ocupación humana del valle del río Bote luego de este evento es escasa y proviene únicamente del sitio Río Bote 2, con fechados hacia ca. 2.030 años AP y ca. 1.010 años AP (Franco et al. 2018). Para estos momentos, en el área se da lo que se conoce como la Anomalía Climática Medieval - ACM - (Stine, 1994 y Tonello 2009 en Franco et al. 2018), durante la cual existió un abandono de este espacio y/o un cambio en su forma de utilización (Borrero \& Franco, 2000; Franco et al. 2016, 2018). Las evidencias recientes indican en este momento la existencia de un cambio genético, además del cultural (Franco et al. 2017b). Si bien durante la ACM se registró un aumento en la humedad en la estepa, RB1 recién vuelve a presentar evidencias de uso hacia ca. 350 años AP. Este fechado cae dentro de lo que se conoce como la Pequeña edad de hielo (LIA, por sus iniciales en inglés). Ésta se manifestó en el Lago Argentino como un periodo de condiciones significativamente más áridas que las actuales, mientras en la estepa se trató de un periodo de mayor humedad (Franco et al. 2018). Sin embargo, para el momento en que RB1 registra evidencias humanas -hace ca. 350 años AP nuevamente se registran condiciones más áridas en la estepa (Tabla 1), (Aniya, 2013 y Strelin et al. 2014 en Franco et al. 2018).

En resumen, las investigaciones realizadas indican que RB1 habría sido usado al menos de dos formas diferentes por los grupos humanos. Durante los momentos más tempranos de la secuencia las actividades llevadas a cabo en el sitio parecen relacionarse únicamente con la subsistencia. Posteriormente, luego de la erupción del volcán Aguilera, RB1 cambia su funcionalidad, usándose repetidamente como lugar de entierro de cuerpos humanos. Finalmente, luego de un hiato, RB1 da cuenta de una ocupación final aislada (Franco et al. 2010, 2017a, 2018). 
Tabla 1. Síntesis de las principales características ambientales y de las ocupaciones humanas en los distintos momentos de ocupación de Río Bote 1, con fechados calibrados.

\begin{tabular}{|c|c|c|}
\hline FECHADOS & AMBIENTE & OCUPACIONES HUMANAS \\
\hline $\begin{array}{l}\text { ca. } 4.800 \text { años AP } \\
\text { (ca. } 5.500 \text { años cal. AP) }\end{array}$ & $\begin{array}{l}\text { Aumento de la humedad en los Andes y } \\
\text { disminución de la humedad en la estepa }\end{array}$ & Exploración inicial \\
\hline $\begin{array}{l}\text { ca. } 4.300 \text { años AP } \\
\text { (ca. } 4.900-4.600 \text { cal. AP) }\end{array}$ & Nueva disminución de la humedad en la estepa & $\begin{array}{l}\text { Mayor evidencia de ocupación al este y } \\
\text { oeste, ocupación efectiva del espacio }\end{array}$ \\
\hline $\begin{array}{l}\text { ca. 4.030-3.860 años AP } \\
\text { (ca. 4.566-4.091 años } \\
\text { cal. AP) }\end{array}$ & $\begin{array}{l}\text { Periodo de condiciones áridas en la zona del } \\
\text { valle de río Bote }\end{array}$ & $\begin{array}{l}\text { Para fines de este periodo: primeros } \\
\text { entierros humanos en abrigos rocosos }\end{array}$ \\
\hline $\begin{array}{l}\text { ca. } 3.400 \text { años AP } \\
\text { (3.500 años cal. AP) }\end{array}$ & $\begin{array}{l}\text { Aumento de la humedad al oeste y condiciones } \\
\text { extremadamente áridas en la estepa }\end{array}$ & Abandono de Río Bote 1 \\
\hline ca. 2.500 cal. AP & $\begin{array}{l}\text { Condiciones más áridas en la zona andina y } \\
\text { leve aumento de la humedad en la estepa }\end{array}$ & Entierro aislado en Río Bote 1 \\
\hline $\begin{array}{l}\text { ca. } 1.900-1.600 \text { años } \\
\text { cal. AP y } 1.500-900 \text { años } \\
\text { cal. AP }\end{array}$ & $\begin{array}{l}\text { Condiciones húmedas en los andes y } \\
\text { condiciones más áridas en la estepa }\end{array}$ & $\begin{array}{l}\text { Periodos centrales de ocupación de las } \\
\text { inmediaciones del lago Argentino }\end{array}$ \\
\hline ca. $1.000-700$ cal. AP & $\begin{array}{l}\text { Anomalía climática medieval: periodo árido en } \\
\text { los Andes y mayor humedad en la estepa }\end{array}$ & $\begin{array}{l}\text { Sitios a cielo abierto en los alrededores del } \\
\text { lago Argentino }\end{array}$ \\
\hline ca. 600 años cal. AP & $\begin{array}{l}\text { Pequeña edad de hielo: Aridez al oeste del lago } \\
\text { Argentino y mayor humedad en la estepa }\end{array}$ & $\begin{array}{l}\text { Cambio en forma de utilización del área o } \\
\text { la presencia de nuevos grupos humanos }\end{array}$ \\
\hline
\end{tabular}

\section{METODOLOGÍA}

Debido a los objetivos que nos propusimos abordar, en este trabajo adquiere una relevancia particular el estudio de los conjuntos desde una perspectiva tafonómica detallada que contribuya a comprender los procesos de preservación y cómo ellos afectan la información contenida en el registro. Para comparar las similitudes y diferencias a través del tiempo, estudiamos cuatro conjuntos zooarqueológicos que provienen de la división de la secuencia estratigráfica en cuatro bloques temporales, definidos según sus características paleoclimáticas y antecedentes arqueológicos. Estos bloques temporales son: 1) Primer bloque: ca. 4.200-4.100 años AP; 2) Segundo bloque: ca. 4.100-3.900 años AP; 3) Tercer bloque: ca. 3.800-3.600 años AP y 4) Cuarto bloque: ca. 350 años AP.

El análisis de cada uno de los cuatro conjuntos faunísticos fue realizado siguiendo los criterios metodológicos propuestos por Mengoni Goñalons (1999). Para cada espécimen se consignó taxón, elemento, segmento, lateralidad y estado de fusión (Mengoni Goñalons, 1999; Kaufmann, 2009). Cuando los rasgos no fueron los suficientemente claros como para realizar una identificación anatómica y taxonómica se asignaron a categorías más inclusivas como Artiodactyla (posible guanaco), Mammalia mediana (animales de entre 10 y $50 \mathrm{~kg}$ ) y Mammalia pequeña (animales de menos de 10 kg) (De Nigris, 2004). Para el análisis cuantitativo de los restos se contemplaron diferentes estimadores de abundancia anatómica y taxonómica comúnmente utilizados en zooarqueología: Número de Especímenes Identificados (NSP), Número de Especímenes No Identificado (NID), Número de Especímenes Identificados por Taxón (NISP), Número Mínimo de Elementos (MNE) y Número Mínimo de Individuos (MNI) (Grayson, 1984; Lyman, 1994a, 2008; Mengoni Goñalons, 1999, 2010). Se calculó la abundancia relativa de partes esqueletarias para el guanaco por ser la especie más representada del sitio, mediante el MAU (Número Mínimo de Unidades Anatómicas) y el \%MAU (Binford, 1981; Lyman, 1994a, 2008). Finalmente, se calculó la relación NSP/NISP para indagar sobre la fragmentación total de cada conjunto (Lyman, 1994a).

Como agentes formadores del registro consideramos tanto a los agentes antrópicos como a los no antrópicos. Para su reconocimiento analizamos los patrones de modificación de las superficies óseas tanto a ojo desnudo como con aumentos que van entre 10X y 30X, ya que a partir de ellos es posible inferir los distintos 
agentes y procesos tafonómicos involucrados en la formación del conjunto y comprender, a partir de este análisis, la historia de formación de un sitio (entre otros, Johnson, 1985; Lyman 1994b). De esta manera, entonces, es posible evaluar el grado de incidencia de los diferentes procesos en la formación de depósitos arqueológicos, el papel jugado por los humanos y los estados de preservación de los conjuntos óseos. Para ello, se obtuvo el perfil de meteorización de los conjuntos óseos (Behrensmeyer, 1978; Fernández-Jalvo, \& Andrews 2016). También se identificó la presencia de termoalteración - Calcinado blanco/gris azulado, carbonizado total negro, parcialmente carbonizado (cuando el porcentaje de termoalteración es menor al $50 \%$ de la superficie ósea) y quemado rojo- (Johnson, 1989; Cain, 2005), de depósitos de óxido de manganeso y de carbonato de calcio (Gutiérrez, 2004; López-González et al. 2006; Marín Arroyo et al. 2008). Asimismo, se registraron marcas de raíces, carnivoros -perforaciones, hoyuelos, arrastre, surcos-, roedores y humanos (Morlan, 1980; Shipman, 1981; Lyman, 2008; Montalvo, 2002; Borrero et al. 2005; Gifford-González, 2018). En el caso del guanaco, por ser la especie más representada, además se distinguieron distintos tipos de huellas antrópicas: corte, machacado, raspado, negativos de impacto y lascados (Binford, 1978, 1981; Enloe, 1993; Gifford-González, 1989, 2018; Mengoni Goñalons, 1999). A partir de su localización se realizaron inferencias sobre las posibles actividades de procesamiento que las habrian originado (entre otros, Binford, 1981; Enloe, 1993; Mengoni Goñalons, 1999). Se tuvo en cuenta el estado de las fracturas -frescas, no-frescas-, el tipo de fractura -longitudinal, transversal, helicoidal- (por ejemplo, Lyman, 1994b, 2008; Mengoni Goñalons, 1999; Gifford-González, 2018). También se evaluó si dichas fracturas presentaban atributos diagnósticos del accionar antrópico asociados, es decir con o sin marcas de impacto y/o percusión asociadas (entre otros, Binford, 1978, 1981; Johnson, 1985; Gifford-González, 1989, 2018).

Finalmente, se analizó la relación entre las partes anatómicas representadas de guanaco y la densidad mineral ósea (DMO) de cada elemento (Elkin, 1995) mediante el uso de un programa estadístico de libre acceso (PAST-Paleontological
Statics). De este modo, se analizó en qué medida la presencia de las partes esqueletarias se debió a la supervivencia diferencial de aquellos elementos con mayor densidad. Debido a que en este trabajo se considera que el resultado de este índice puede estar sesgado por las propiedades óseas y la elección de la unidad de análisis, se decidió adoptar la propuesta metodológica utilizada por De Nigris y Mengoni Goñalons (1999), la cual considera todo el esqueleto y las diferentes regiones por separado, como unidades analíticas diferentes.

\section{RESULTADOS}

Se sintetizan a continuación los resultados alcanzados por bloque temporal.

\section{Primer bloque (ca. 4.200 a 4.100 años AP)}

El conjunto está compuesto por un total de 1139 especímenes óseos, de los cuales el $43,5 \% \quad(\mathrm{NISP}=496)$ pudo ser identificado taxonómicamente. Un 66,4\% ( $\mathrm{n}=427)$ del NID posee fracturas en estado fresco y la relación $\mathrm{NSP} / \mathrm{NISP}=2,29$ da cuenta de un conjunto medianamente fragmentado. Si bien el guanaco (Lama guanicoe) es la especie que predomina en el conjunto $(67,7 \%$; $n=336)$, otras categorías taxonómicas también se encuentran representadas, aunque en porcentajes menores a $17,5 \%$, entre ellas aves, roedores y carnívoros (Tabla 2).

\section{Representación anatómica del guanaco}

En cuanto a la representación anatómica del guanaco, predomina el esqueleto apendicular $85,5 \%(n=278)$ frente al axial $14,5 \%(n=47)$, sin considerar los fragmentos de dientes $(n=11)$. Si bien la frecuencia de especímenes de la parte axial del esqueleto es baja, ésta se encuentra representada por casi todos los elementos que la componen. Por su parte todos los elementos de la porción apendicular se encuentran presentes (Fig. 3).

En el conjunto predominan los elementos cuyas epífisis se encuentran fusionadas $(57,6 \%)$; sin embargo, se ha registrado un número alto de elementos con epífisis sin fusionar $\mathrm{y} / \mathrm{o}$ semifusionadas (18,8\%), mientras que en el $23,6 \%$ de los casos no fue posible determinar el 
Tabla 2. Determinación taxonómica por bloque temporal.

Referencias: Indet. =indeterminado, peq. $=$ pequeño $/ \mathrm{a}$, med. $=$ mediano $/ \mathrm{a}$

\begin{tabular}{|c|c|c|c|c|c|c|c|c|}
\hline \multirow{2}{*}{ Taxón } & \multicolumn{2}{|c|}{ Primer Bloque } & \multicolumn{2}{|c|}{ Segundo Bloque } & \multicolumn{2}{|c|}{ Tercer Bloque } & \multicolumn{2}{|c|}{ Cuarto Bloque } \\
\hline & NISP (\%) & MNE & NISP (\%) & MNE & NISP (\%) & MNE & NISP (\%) & MNE \\
\hline Liolaemus indet. & 0 & 0 & 0 & 0 & $2(0,3 \%)$ & 2 & $3(2,3 \%)$ & 2 \\
\hline Ave indet. peq. & $30(6 \%)$ & 25 & $13(1,5 \%)$ & 4 & $8(1,3 \%)$ & 7 & $2(1,5 \%)$ & 1 \\
\hline Ave indet. med. & 0 & 0 & $3(0,3 \%)$ & 2 & $2(0,3 \%)$ & 1 & $3(2,3 \%)$ & 1 \\
\hline Rhea pennata & $1(0,2 \%)$ & 1 & $8(0,9 \%)$ & 1 & 0 & 0 & 0 & 0 \\
\hline Tacheyres brachypterus & 0 & 0 & $3(0,3 \%)$ & 3 & 0 & 0 & 0 & 0 \\
\hline Mammalia peq. & $5(1 \%)$ & - & $5(0,6 \%)$ & - & 0 & 0 & 0 & 0 \\
\hline Mammalia med. & 0 & 0 & $29(3,3 \%)$ & - & $40(6,5 \%)$ & - & $111(85,4 \%)$ & - \\
\hline Rodentia indet. peq. & $29(5,8 \%)$ & 22 & $45(5,1 \%)$ & 41 & $6(1 \%)$ & 5 & 0 & 0 \\
\hline Rodentia indet. med. & 0 & 0 & 0 & 0 & $6(1 \%)$ & 5 & 0 & 0 \\
\hline Chinchillidae & 0 & 0 & 0 & 0 & $2(0,3 \%)$ & 2 & 0 & 0 \\
\hline Lagidium sp. & $1(0,2 \%)$ & 1 & $1(0,1 \%)$ & 1 & 0 & 0 & 0 & 0 \\
\hline Conepatus humboldtii & 0 & 0 & $3(0,3 \%)$ & 3 & 0 & 0 & 0 & 0 \\
\hline Lycalopex griseus & $7(1,4 \%)$ & 7 & $8(0,9 \%)$ & 7 & 0 & 0 & 0 & 0 \\
\hline Lycalopex culpaeus & 0 & 0 & $6(0,7 \%)$ & 5 & 0 & 0 & 0 & 0 \\
\hline Puma concolor & 0 & 0 & $1(0,1 \%)$ & 1 & 0 & 0 & 0 & 0 \\
\hline Artiodactyla indet. & $87(17,5 \%)$ & - & 423 (48\%) & - & $278(45 \%)$ & - & $1(0,8 \%)$ & 1 \\
\hline Ovis orientalis aries & 0 & 0 & 0 & 0 & 0 & 0 & $1(0,8 \%)$ & 1 \\
\hline Lama guanicoe & $336(67,7 \%)$ & 180 & $333(37,8 \%)$ & 142 & $274(44,3 \%)$ & 145 & $9(6,9 \%)$ & 7 \\
\hline Total & 496 & 235 & 881 & 210 & 618 & 167 & 130 & 13 \\
\hline NTAXA & 3 & & 7 & & 2 & & 3 & \\
\hline
\end{tabular}

estado de fusión. De acuerdo con los puntos de fusión propuestos por Kaufmann (2009), entre aquellos elementos que fusionan tempranamente se han encontrado sin fusionar tres especímenes de radio-cúbito distal $(\mathrm{RC} 1)$ y tres especímenes de vértebras lumbares (VL1). Además, se hallaron sin fusionar y semifusionados especímenes pertenecientes a elementos de fusión tardía (más de 19 meses). La estimación del MNI, teniendo en cuenta la lateralidad y los estadios de fusión ósea, indica que un número mínimo de 5 individuos se requiere para dar cuenta de las frecuencias de elementos presentes: uno de éstos pertenecería a un individuo menor a tres meses -sobre la base de los especímenes de vértebras lumbares antes mencionados-, otro correspondería a uno menor a seis meses -teniendo en cuenta la epífisis proximal derecha sin fusionar del radio-cubito- y los tres individuos restantes serían mayores de seis meses -en base a la epífisis proximal izquierda fusionada del radio-cúbito-.
Modificaciones óseas

El análisis de la superficie ósea de los huesos de guanaco da cuenta de un perfil de meteorización desarrollado, ya que se encuentran representados casi todos los estadios (del 0 al 4). Este perfil queda conformado por un $61 \%$ de la muestra en el estadio 0 (no meteorizada), un $25 \%$ en estadios muy bajos ( 1 y 2), y solo un $14 \%$ en estadios avanzados ( 3 y 4), no registrándose especímenes en el estadio 5 (Fig. 4).

Se identificaron especímenes óseos termoalterados en baja frecuencia $(9,2 \%$ del total del conjunto, $n=104)$. El mayor porcentaje de termoalteración corresponde a especímenes parcialmente carbonizados $(6,2 \%, \mathrm{n}=71)$, solo se observó un espécimen quemado $(0,09 \% ; n=1)$ y el porcentaje de especímenes carbonizados y calcinados apenas alcanza el $1 \%$ ( $n=18$ y $\mathrm{n}=15$, respectivamente). Entre los especímenes termoalterados se encuentran fragmentos óseos 


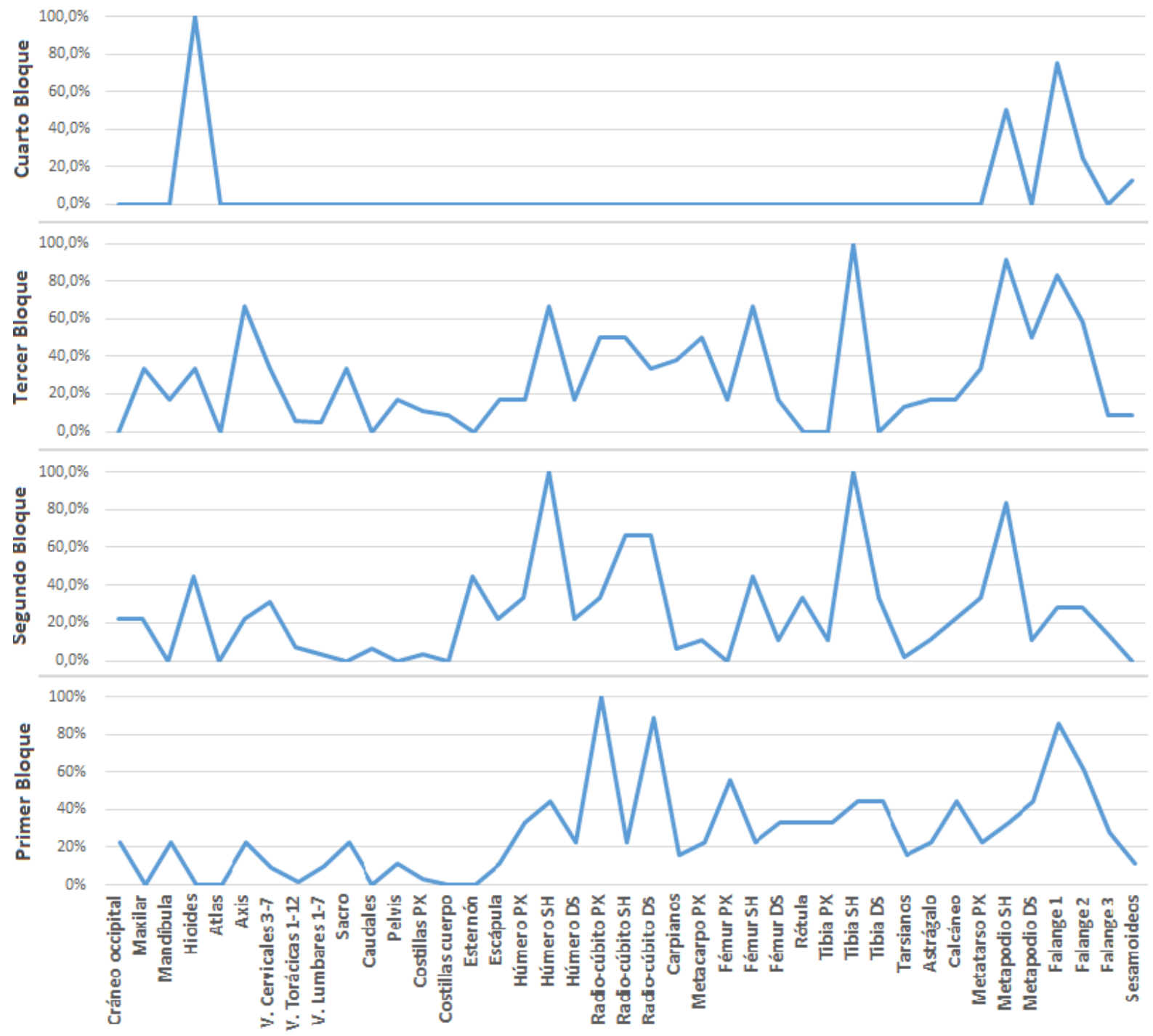

Fig. 3. Frecuencia relativa de Unidades Anatómicas Mínimas (\%MAU) por bloque temporal

de taxón indeterminado, Artiodactyla y guanaco, entre los que se observa la misma tendencia que las del conjunto general, destacándose entre los elementos de esta última especie, la presencia de alteración térmica en fragmentos de epífisis y diáfisis de huesos largos, falanges, vértebras, fragmentos de huesos planos y cráneo.

El óxido de manganeso afecta el $96,6 \%$ $(\mathrm{n}=1101)$ del total del conjunto, expresándose en forma de pequeños puntos y manchones con patrón dendrítico sobre las superficies óseas. El carbonato de calcio se registra en un $83,7 \%$ ( $n=954)$ del conjunto, tomando la forma de manchones pequeños y en algunos casos pequeñas concreciones cristalinas. En ningún caso estas sustancias comprometen más del $10 \%$ de las superficies óseas de los especímenes. Las modificaciones por raíces siguen en importancia, alcanzando un $38 \% \quad(n=434)$ del conjunto, afectando menos del $20 \%$ de las superficies de los especímenes, adquiriendo la forma de finas improntas dendríticas superficiales de color castaño rojizo (Fig. 5).

Las marcas de carnivoros y roedores tienen una representación muy baja, la cual ronda el $1 \%$ ( $n=8$ para carnivoros y $n=12$ para roedores; 


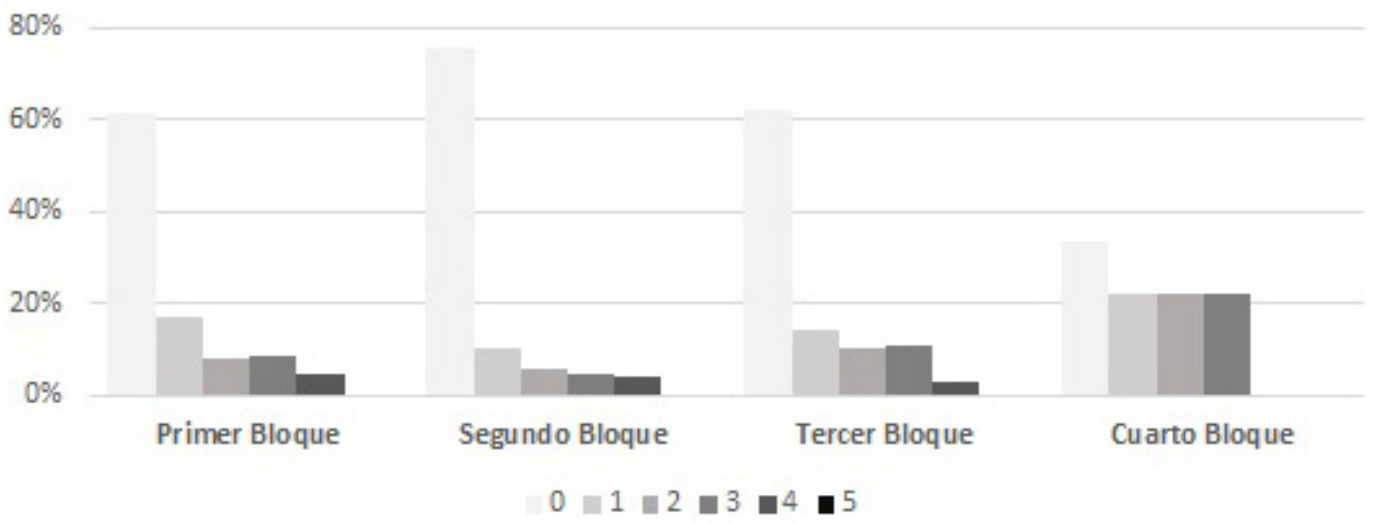

Fig. 4. Perfil de meteorización por bloque temporal.

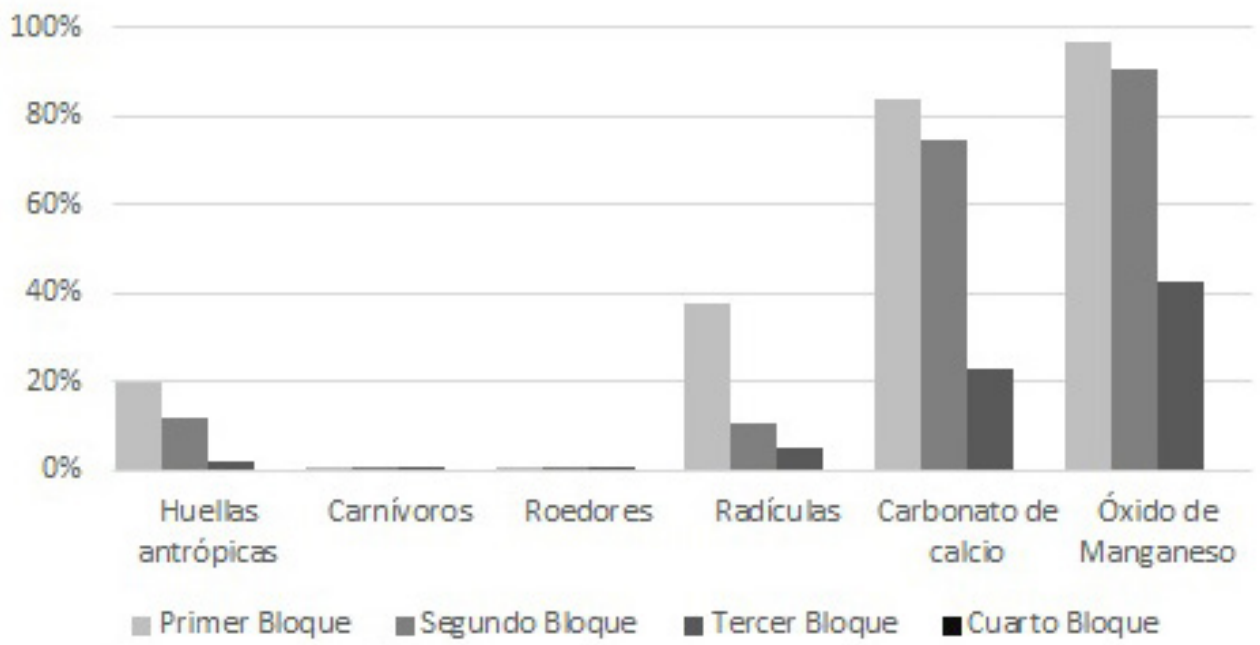

Fig. 5. Frecuencia relativa de modificaciones óseas por bloque temporal.

Fig. 5). Las primeras se encuentran sobre huesos largos de guanaco (epífisis y diáfisis) y también sobre una falange medial y un arco cigomático. Los tipos de huellas de carnívoros identificados corresponden a surcos, perforaciones $y$ hoyuelos. Al menos dos especímenes registran en conjunto marcas de carnívoros y huellas antrópicas no superpuestas. Las marcas de roedor se encuentran mayoritariamente sobre falanges de guanaco, pero también sobre un fragmento de tibia. El resto de los especímenes con huellas de roedor son fragmentos y astillas de Artiodactyla y taxón indeterminado. El tipo de huellas registrado es de surcos paralelos en pares. Cabe mencionar que cinco especímenes presentan en conjunto huellas de roedores $y$ marcas antrópicas no superpuestas.

Las huellas antrópicas son las más representadas en el conjunto, alcanzado un $19,7 \% \quad(n=225)$ del total de los restos óseos (Fig. 5). Se reconocieron sobre fragmentos óseos de taxón indeterminado, choique (Rhea pennata), Artiodactyla y guanaco. Del total de los especímenes de guanaco $(n=325$, excluyendo los dientes y molares), el $42,4 \%(n=138)$ posee huellas de corte y/o percusión. En cuanto al total de las huellas registradas sobre el guanaco, un $6,5 \%(\mathrm{n}=9)$ se reconoció sobre especímenes de 
la porción axial, mientras que el 93,5\% ( $n=129)$ restante se identificó sobre especímenes de la porción apendicular del esqueleto. La mayoría de los elementos poseen huellas antrópicas, a excepción de: el cráneo, el axis, las vértebras cervicales y el sacro - en el esqueleto axialel húmero distal, el metatarso proximal, los tarsianos, la rótula y los sesamoideos -en el esqueleto apendicular-. En cuanto a los tipos de huellas registradas, dominan las huellas de corte que alcanzan el 52,5\% ( $\mathrm{n}=92)$, seguidas por los negativos de impacto $(41,2 \%, \mathrm{n}=72)$, lascados $(5,1 \%, \mathrm{n}=9) \mathrm{y}$, finalmente, raspado $(1,2 \%, \mathrm{n}=2)$. Las huellas de corte se registran tanto en la porción axial como en la apendicular, mientras que el resto de las huellas solo se registran en la porción apendicular (excepto por un negativo de impacto sobre un fragmento de pelvis).

Un 99\% ( $n=1030)$ del total del conjunto se encuentra fragmentado, del cual un $73 \%(n=756)$ se presenta fragmentado en estado fresco. Del total de los especímenes de guanaco ( $\mathrm{n}=325$ sin contar los dientes), un $78,7 \%(n=256)$ se encuentra fragmentado y un $86,7 \%(n=222)$ de éstos presenta fracturas en estado fresco. Estas últimas presentan superficies de fractura suaves y del mismo color que la superficie cortical del hueso. Entre ellas, la mayoría corresponde a patrones helicoidales $(37,2 \%, n=93)$ y longitudinales $(34 \%, n=85)$. Debe destacarse, que un alto porcentaje de especímenes fragmentados en estado fresco también presenta huellas de percusión asociadas (Fig. 6 y 7).

Densidad mineral ósea

La correlación entre el \%MAU total del conjunto y la DMO del guanaco no es estadísticamente significativa ( $r s=0,31 ; p>0,05$ ). $\mathrm{Al}$ desglosar los valores correspondientes tanto a la porción axial ( $r s=-0,18 ; \mathrm{p}>0,05)$ como a la apendicular ( $r s=0,07 ; \mathrm{p}>0,05)$, tampoco se encontraron valores significativos que den cuenta de que el perfil del conjunto estudiado estuviese afectado por este tipo de procesos.

\section{Segundo bloque (ca. 4.100 a 3.900 años AP)}

Este conjunto está compuesto por un total de 2642 especímenes óseos, de los cuales el $33,3 \% \quad(\mathrm{NISP}=881)$ pudo ser identificado taxonómicamente. Entre los NID, el 73\% $(n=1289)$ se encuentra fracturado en estado fresco. La relación NSP/NISP $=2,99$ da cuenta de un conjunto levemente más fragmentado en comparación con el bloque previo. Como ya fue mencionado, en este bloque temporal se registró la presencia de un instrumento realizado sobre un resto óseo indeterminado (Franco et al. 2017 b). Dominan los especímenes asignados al orden Artiodactyla (48\%; $\mathrm{n}=423$ ); sin embargo, la especie más representada continúa siendo el guanaco $(37,8 \% ; n=333)$. Respecto al bloque temporal previo, aquí se observa un aumento en el número de especies identificadas, aunque ellas se encuentran en porcentajes inferiores al $1 \%$ (Tabla1).

Representación anatómica del guanaco

En cuanto a la representación anatómica del guanaco, sin considerar los dientes $(n=12)$, domina la porción apendicular $(55,5 \% ; n=178)$ frente a la porción axial $(44,5 \% ; n=143)$. Esta última se encuentra representada por pocos elementos, mientras que en la porción apendicular se registran casi todos los elementos que la componen. A diferencia del bloque previo, se distingue un patrón claro de dominancia de diáfisis sobre las epífisis de los huesos largos (Fig. 3).

$\mathrm{Al}$ igual que en el bloque previo, en este conjunto dominan los elementos cuyas epífisis se encuentran fusionadas (51,9\%). Sin embargo, también se han registrado elementos con epífisis sin fusionar y/o semifusionadas (18\%), finalmente en el $30,1 \%$ restante no fue posible determinar el estado de fusión. Entre aquellos elementos que fusionan tempranamente se han encontrado sin fusionar cinco especímenes de radio-cúbito distal (RC1)y cuatro especímenes de metapodio proximal (MC2/MT2). Otros especímenes sin fusionar y semifusionados también se encuentran presentes, todos pertenecientes a elementos de fusión tardía (más de 19 meses). El cálculo del MNI, teniendo en cuenta la lateralidad y los estadios de fusión, da cuenta de un mínimo de cuatro individuos, entre los cuales habría un individuo menor a seis meses (sobre la base del radio-cúbito distal derecho sin fusionar) otro sería menor a tres meses (presencia 
de un metapodio proximal sin fusionar) y los últimos dos corresponderían a individuos mayores a 48 meses (en base a la epífisis proximal del radio-cubito fusionada).

\section{Modificaciones óseas}

Este bloque también presenta un perfil desarrollado de meteorización de los restos óseos de guanaco, representado por casi todos los estadios (del 0 al 4). Sin embargo, el $76 \%$ de la muestra se encuentra en el estadio 0 (no meteorizado), el 16\% se presenta en estadios muy bajos de meteorización (1 y 2), y solo el $8 \%$ en estadios avanzados (3 y 4), (Fig. 4).

La frecuencia de especímenes termoalterados en todo el conjunto es muy baja $(13 \%, n=354)$. Entre ellos se registraron ejemplares parcialmente carbonizados $(6,4 \%$; $\mathrm{n}=175)$, totalmente carbonizados $(4,6 \%, \mathrm{n}=125)$ y calcinados $(2 \%, n=54)$. Se hallaron fragmentos termoalterados de taxón indeterminado, ave pequeña, cáscara de huevo de choique, Mammalia mediana, Artiodactyla y guanaco. Si desglosamos los valores obtenidos para el guanaco, éstos mantienen el mismo perfil que el conjunto completo. Entre los restos óseos termoalterados de guanaco se registraron fragmentos de epífisis y diáfisis de huesos largos, fragmentos de huesos planos, falanges, vértebras y fragmentos de huesos de la cabeza como el hioides y el cráneo.

Los depósitos de óxido de manganeso afectan al 90,6\% ( $n=2394)$ del conjunto, expresándose en forma de pequeños manchones de patrón dendrítico. El carbonato de calcio afecta un $75,8 \%(n=2003)$ de la muestra, tomando la forma de manchones pequeños $y$, en algunos casos, pequeñas concreciones cristalinas. El tercer agente modificador son las raíces, afectando tan solo un $11 \%(n=292)$ de la muestra. Éstas se presentan en forma de finas improntas dendríticas, de color castaño rojizo. En este bloque temporal todas las sustancias adheridas mencionadas afectan menos del 10\% de las superficies óseas (Fig. 5).

Las marcas de carnivoros y roedores se presentan en porcentajes muy bajos $(0,41 \%$; $\mathrm{n}=11$ y $0,11 \% ; n=3$, respectivamente; Fig. 5). Las primeras se registran sobre fragmentos de diáfisis de huesos largos de guanaco y Artiodactyla. El tipo de huella identificado corresponde a surcos y hoyuelos. Las marcas de roedor se encuentran sobre un calcáneo y una diáfisis de radio-cúbito de guanaco y sobre una diáfisis de Artiodactyla. El tipo de marcas registrado es de surcos paralelos en pares. Solo se registró la presencia conjunta de marcas no superpuestas de carnívoro, roedor y huellas antrópicas en la diáfisis de un radio-cúbito.

En este bloque las huellas antrópicas alcanzan un $12,8 \%$ ( $n=339$; Fig. 5). Éstas se registraron sobre fragmentos óseos de taxón indeterminado, ave pequeña y mediana, pato vapor malvinero, zorro gris, Artiodactyla y guanaco. Cabe mencionar que en el caso del pato vapor malvinero, se trata de dos diáfisis cuyos extremos proximales han sido removidos.

Del total de los especímenes de guanaco ( $n=321$, excluyendo los dientes y molares), el $32,7 \%$ ( $n=105)$ presenta huellas de corte $y / 0$ percusión. De ellas, el 12,5\% ( $n=19)$ se registró en especímenes de la porción axial, mientras que el $87,5 \%$ ( $n=137)$ restante se identificó en elementos de la porción apendicular. Si bien la mayoría de los elementos registran la presencia de huellas antrópicas, existen excepciones como: el axis, vértebras cervicales, lumbares y caudales en el esqueleto axial; y el húmero distal, fémur distal, tibia proximal y distal, metatarso proximal, carpianos y tarsianos, en el caso del esqueleto apendicular. En cuanto a los tipos de huellas registradas, dominan los especímenes que presentan negativos de impacto ( $25 \%$; $n=75$ ), seguidos por aquellos con huellas de corte $(18 \% ; n=56)$, lascados $(8 \% ; n=23)$, machacado $(1 \% ; n=3)$ y, finalmente, raspado $(1 \%$; $\mathrm{n}=2$ ). Los negativos de impacto, huellas de corte y lascados se registran tanto en la porción axial como en la apendicular, mientras las huellas de machacado y raspado solo están presentes en la porción apendicular. Existe un claro patrón de dominancia de las huellas antrópicas sobre las diáfisis de los huesos largos, tanto de corte como de percusión, aunque estas últimas en mayor número.

Un 98\% ( $n=2599)$ del total del conjunto se encuentra fragmentado, del cual un $74,2 \%$ $(n=1963)$ se presenta fragmentado en estado fresco. Del total de los especímenes de guanaco ( $n=321$ sin contar los dientes) un $96 \%(n=307)$ se encuentra fragmentado y un $77 \%(n=237)$ presenta fracturas en estado fresco (Fig. 6 y 7). En el 81,4\% 
( $n=261$ ) de los casos, el color de la superficie de fractura fue el mismo que el de la superficie ósea. En su mayoría corresponden a patrones longitudinales (42\%, $n=129)$ y transversales $(39,4 \%, n=121)$, mientras que las fracturas helicoidales alcanzan el $18,5 \%(n=57)$. Además, un alto porcentaje de especímenes fragmentados en estado fresco también presenta huellas de percusión asociadas.

Densidad mineral ósea

La correlación entre el \%MAU del guanaco y la densidad mineral ósea de dicha especie es positiva, moderada y estadísticamente significativa $(r s=0,46 ; p<0,05)$, alertando sobre un posible caso de supervivencia diferencial. Sin embargo, la correlación \%MAU-DMO en el esqueleto axial es negativa, moderada y estadísticamente no significativa ( $r s=-0,36 ; p>0,05)$; en cambio, la correlación \%MAU-DMO apendicular es positiva, media y estadísticamente significativa ( $r s=0,53$; $\mathrm{p}<0,01)$.

\section{Tercer bloque (ca. 3.800 a 3.600 años AP)}

Este bloque contiene un total de 4019 especímenes, de los cuales el 15\% (NISP=618) pudo ser identificado taxonómicamente. Un $25 \%$ $(n=878)$ de NID se encuentra fracturado en estado fresco. La relación NSP/NISP=6,59 da cuenta de un conjunto altamente fragmentado, más del doble que los dos bloques temporales previos. Entre los especímenes identificados a nivel taxonómico dominan los adjudicados al orden de Artiodactyla (45\%; $\mathrm{n}=278)$, mientras que la especie más representada sigue siendo el guanaco (44\%, $\mathrm{n}=274$ ). Otros taxones se encuentran presentes, pero siempre en porcentajes menores al 6,5\%. En este bloque se registra una disminución de los taxones representados respecto del bloque anterior (Tabla 2).

Representación anatómica del guanaco

En cuanto al perfil anatómico del guanaco, domina la porción apendicular $(76,5 \% ; n=183)$ frente a la porción axial $(23,5 \% ; n=55)$ del esqueleto, sin contar los dientes $(n=36)$. La porción axial se encuentra representada por casi todos los elementos que la componen, con excepción del atlas, las vértebras caudales y el esternón. La porción apendicular, a diferencia de los bloques previos, se encuentra representada por un número menor de elementos (no se encuentran presentes la rótula y las porciones proximales y distales de la tibia). Al igual que en el segundo bloque, se distingue un patrón claro de dominancia de diáfisis sobre las epífisis de los huesos largos (Fig. 3).

En este conjunto dominan los elementos cuyas epífisis se encuentran fusionadas (54\%); sin embargo, también se han registrado elementos con epífisis sin fusionar $y / 0$ semifusionadas $(12,6 \%)$, mientras que en el $33,4 \%$ de los casos no fue posible determinar el estado de fusión. Entre aquellos elementos que fusionan tempranamente, se han encontrado sin fusionar una epífisis proximal y otra distal de radio-cubito ( $\mathrm{RC} 1 \mathrm{y}$ RC6), y una epífisis proximal de metacarpo (MC2). Otros especímenes sin fusionar y semifusionados también están presentes, todos pertenecientes a elementos de fusión tardía (más de 19 meses). El cálculo del MNI, teniendo en cuenta la lateralidad y los estadios de fusión ósea, da cuenta de un mínimo de 3 individuos. Este número mínimo fue calculado teniendo en cuenta la presencia de un individuo menor a 3 meses de edad (sobre la base de la presencia de un radio-cúbito proximal derecho sin fusionar) y dos individuos mayores a tres meses de edad (en base a la epífisis proximal derecha del radio-cubito fusionada).

\section{Modificaciones óseas}

Este bloque presenta un perfil desarrollado de meteorización del guanaco, encontrándose representados los estadios del 0 al 4 . Sin embargo, el $62 \%$ de la muestra se encuentra en el estadio 0 (no meteorizada), el $24 \%$ en estadios muy bajos (1 y 2) y el $14 \%$ en estadios avanzados (3 y 4) (Fig. 4).

La frecuencia de especímenes termoalterados de todo el conjunto es muy baja, alcanzado el $11,3 \%(n=455)$. Se registró solo un $0,04 \%(n=2)$ de especímenes quemados (marrónrojo con superficie lustrosa), un $3,6 \% \quad(n=146)$ parcialmente carbonizado en un extremo, un 7,5\% $(\mathrm{n}=294)$ totalmente carbonizado y tan solo un $0,3 \%(n=13)$ de especímenes calcinados. En este bloque se encontraron fragmentos termoalterados 
de especímenes óseos de taxón indeterminado, Mammalia mediana, Artiodactyla y guanaco. Si separamos los valores obtenidos para el guanaco, este mantiene el mismo perfil. Entre los restos óseos termoalterados de guanaco se registraron fragmentos de epífisis y diáfisis de huesos largos, falanges y fragmentos de vértebras.

El óxido de manganeso afecta un $43 \%$ $(n=1727)$ del conjunto, en forma de pequeños puntos con patrón dendrítico. Tan solo el $23 \%$ $(n=917)$ de los restos óseos presenta carbonato de calcio, estos toman forma de manchones pequeños $y$ en muy pocos casos, pequeñas concreciones cristalinas. El tercer agente modificador identificado son las raíces, las cuales afectan solo un $5 \%(n=217)$ del conjunto, presentándose en forma de improntas dendríticas superficiales de color castaño rojizo y en forma de radículas aún adheridas al hueso. En este bloque dichas modificaciones afectan menos del $10 \%$ de las superficies de los especímenes (Fig. 5).

Las marcas de carnivoro y roedores se presentan en porcentajes muy bajos $(0,05 \%$; $\mathrm{n}=2$ y $0,15 \% ; \mathrm{n}=6$, respectivamente; Fig. 5). Las primeras se encuentran sobre fragmentos de diáfisis de huesos largos de Artiodactyla. El tipo de marca identificado corresponde a surcos y hoyuelos. Las marcas de roedor se encuentran en su mayoría sobre epífisis de falanges y huesos largos de guanaco y Artiodactyla ( $n=4$ y $n=1$, respectivamente), aunque también sobre un fragmento de fémur de roedor mediano $(n=1)$. El tipo de huellas registrado es de surcos paralelos en pares. Cabe mencionar que solo una diáfisis distal de metatarso de guanaco presenta en conjunto huellas de roedores y huellas de corte, no encontrándose superpuestas.

En este bloque temporal se registró un muy bajo porcentaje de especímenes con huellas antrópicas, constituyendo tan solo un 2,4\% ( $n=99)$ del total de conjunto (Fig. 5). Estas se identificaron sobre fragmentos óseos de especímenes indeterminados, Mammalia mediana, Artiodactyla y guanaco.

Solo el 25,4\% ( $n=61)$ del total de los especímenes de guanaco $(n=240$, excluyendo los dientes y molares), posee huellas de corte y/o percusión. El $8 \%(n=5)$ se registró sobre su porción axial, mientras que el $92 \%(n=56)$ restante se localizó sobre la porción apendicular. En cuanto a los tipos de marcas antrópicas, dominan los especímenes con huellas de corte (14\%; $n=34)$, seguidas por los negativos de impacto $(9 \% ; n=22)$, lascados $(1 \% ; n=4)$ y marcas de machacado $(0,01 \% ; n=1)$. Es interesante destacar la ausencia de marcas de raspado en este conjunto, las que si se encuentran presentes en los dos bloques temporales previos. Las huellas de corte se registran tanto en la porción axial como en la apendicular del esqueleto, mientras que las huellas de percusión solo se encuentran presentes en especímenes de la porción apendicular, principalmente sobre las extremidades medias e inferiores. Existe un claro patrón de dominancia de huellas de corte sobre las diáfisis de todos los huesos largos y sobre la epífisis proximal del metatarso y metacarpo, siguiendo en importancia aquéllas presentes sobre la epífisis distal del radio-cúbito. En el caso de las huellas de percusión, las frecuencias más elevadas se encuentran en las porciones distales de las extremidades medias e inferiores, con la excepción de la diáfisis del húmero.

Un 98,8\% ( $n=3974)$ del total del conjunto se encuentra fragmentado, del cual tan solo un $28,3 \% \quad(n=1125)$ se presenta fragmentado en estado fresco. Del total de los especímenes de guanaco ( $n=238$ sin contar los dientes), un $85,2 \%$ $(n=203)$ se encuentra fragmentado (Fig. 6 y 7). A diferencia de los bloques temporales anteriores, en el total del conjunto dominan, en general, las fracturas en estado seco (un 63\%, n=128, de los fragmentos de huesos de guanaco). Éstas en su mayoría presentan una coloración distinta a la superficie ósea, con patrones transversales astillados o irregulares $(47,3 \%$ de la muestra con fracturas en estado seco, $n=61$ ) y longitudinales astillados $(30,5 \%, n=39)$, mientras que el $22,2 \%$ $(n=28)$ restante son fracturas longitudinales $y$ transversales. Por otro lado, las fracturas en estado fresco $(37 \%, n=75$, del total de los especímenes fragmentados) son en su mayoría helicoidales $(52 \%, n=39)$ y longitudinales $(45 \%, n=34)$. Las diáfisis de los huesos largos son las únicas porciones sobre las que dominan las fracturas en estado fresco (69\% de este conjunto). Más allá del bajo porcentaje de especímenes con fracturas en estado fresco del conjunto total, un alto porcentaje de éstas registra una elevada frecuencia relativa de huellas de percusión. 
Densidad mineral ósea

La correlación entre el \%MAU y el índice de densidad mineral ósea del guanaco es positiva, moderada y estadísticamente significativa ( $r s=0,37$; $p<0,05)$. Sin embargo, si desglosamos el resultado obtenido para la totalidad del esqueleto del guanaco, teniendo en cuenta las distintas porciones de éste, la correlación \%MAU-DMO en el esqueleto axial es positiva, muy cercana a cero y estadísticamente no significativa ( $r s=0,06 ; p>0,05)$, mientras que la correlación \%MAU-DMO apendicular es positiva, media-alta y estadísticamente significativa ( $r s=0,69$; $\mathrm{p}<0,01)$.

\section{Cuarto bloque (ca. 350 años AP)}

Este conjunto está compuesto por un total de 190 especímenes, deloscualesel68,5\%(NISP =130) pudo ser identificado taxonómicamente. Tan solo un $16 \%(n=10)$ del NID se encuentra fracturado en estado fresco. La relación NSP/NISP $=1,46$ da cuenta de un conjunto menos fragmentado que los depósitos previos. La categoría más amplia de Mammalia mediana es la que predomina $(85,4 \%$; $\mathrm{n}=111)$; sin embargo, la especie más representada continúa siendo el guanaco $(6,9 \% ; n=9)$. Otros taxones se encuentran representados, aunque siempre en porcentajes menores al 2,3\% (Tabla 2). Debe destacarse que por primera vez en el sitio aparece fauna introducida identificada a partir de un fragmento de metapodio semifusionado de Ovis orientalis aries.

\section{Representación anatómica del guanaco}

En cuanto a la representación anatómica del guanaco, domina el esqueleto apendicular $(88,8 \%$; $\mathrm{n}=8$ ) frente al axial (11,2\%; $\mathrm{n}=1$ ), (Fig. 3). Debe considerarse, antes de continuar con el análisis, que la muestra proveniente de este bloque es pequeña, por tal motivo las interpretaciones realizadas a partir del material del cuarto bloque pueden llegar a ser poco confiables.

La estimación del MNI, teniendo en cuenta la lateralidad y los estadios de fusión ósea, indica que un número mínimo de 2 individuos se requiere para dar cuenta de las frecuencias de elementos presentes. Uno de estos individuos fue definido sobre la base de la presencia de una epífisis proximal derecha sin fusionar correspondiente a una falange primera (la cual sugiere la presencia de un individuo menor a 30 meses), mientras que el segundo individuo fue definido sobre la base de la presencia de una epífisis proximal izquierda fusionada del mismo elemento (la cual indica la presencia de al menos un individuo mayor de 30 meses). En el conjunto predominan los elementos cuyas epífisis se encuentran fusionadas, con las excepciones antes referidas en la estimación de la edad de los individuos.

\section{Modificaciones óseas}

Este bloque presenta un perfil poco desarrollado de meteorización (estadios del 0 al 3). Sin embargo, a diferencia de lo que ocurre en los bloques previos, en este depósito solo el 33\% de la muestra no se encuentra meteorizado (estadio $0)$, mientras que un $44 \%$ se encuentra en estadios muy bajos de meteorización (1 y 2) y un $23 \%$ en estadios avanzados (3), (Fig. 4).

Si bien se identificaron especímenes óseos termoalterados, su frecuencia es muy baja (menos del $10 \%$ de todo el conjunto). El mayor porcentaje de termoalteración corresponde a especímenes parcialmente carbonizados $5,4 \%(n=11)$, mientras que el porcentaje de calcinados apenas alcanza el $3,5 \%(n=7)$. En este conjunto, solo los especímenes de taxón indeterminado y aquéllos identificados como Mammalia mediana presentan evidencias de termoalteración.

Este bloque no presenta evidencia de depósitos de carbonato de calcio ni de óxido de manganeso sobre los restos óseos. Tampoco se registraron marcas de raíces, de carnívoros, ni de roedores. Para finalizar, no se encontró evidencia de huellas antrópicas (ni de corte, ni de percusión) sobre ningún espécimen.

Un 98,9\% ( $\mathrm{n}=180)$ del total del conjunto se encuentra fragmentado, del cual tan solo un $17 \%$ $(n=32)$ se presenta en estado fresco. Un $78 \%(n=7)$ de los especímenes de guanaco se encuentran fragmentados, de los cuales un $57 \%(n=4)$ se encuentra en estado fresco. El tipo de fractura en estado fresco que domina es la longitudinal (57\%; $\mathrm{n}=4)$. Sin embargo, como ya fue mencionado, en este depósito no hay huellas de percusión 


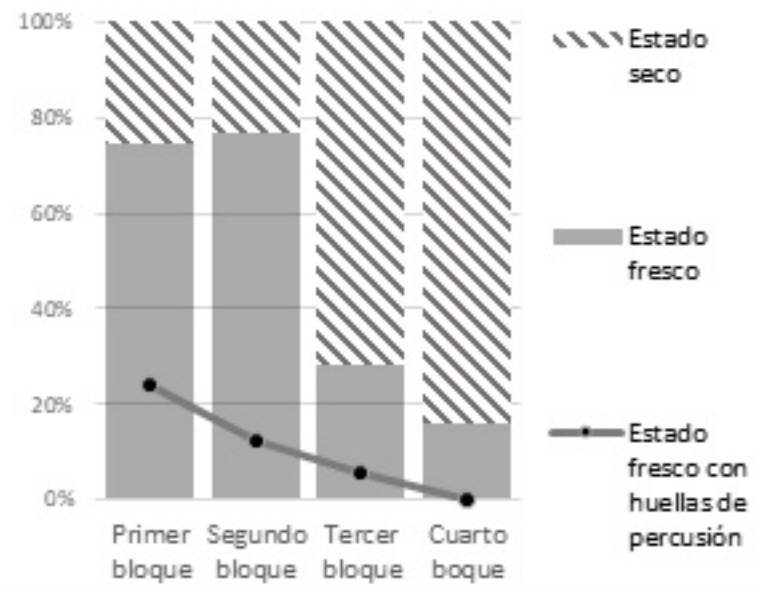

Fig. 6. Estado de fragmentación del NSP y huellas antrópicas asociadas.

asociadas a las fracturas frescas que sugieran la fractura intencional de los restos o algún tipo de procesamiento del guanaco (Fig. 6 y 7).

Densidad mineral ósea

Debido al escaso número de especimenes de guanaco en este bloque temporal, las correlaciones estadísticas como las usadas para visualizar los efectos de la DMO no resultan metodológicamente adecuadas. Sin embargo, podemos afirmar que no se encuentran representados elementos de baja densidad ósea, estando presentes únicamente dos elementos con densidad media-baja (falange proximal y vértebra cervical) y un elemento de densidad mediaalta (tarsiano). Estos datos sugieren que el conjunto podría estar mediado por los efectos de la DMO.

\section{DISCUSIÓN}

Sintetizaremos a continuación las principales semejanzas y diferencias entre conjuntos.

Efectos de la humedad, accionar de raíces, depositación de carbonato de calcio y óxido de manganeso sobre los restos óseos

En primer lugar, como puede observarse en el Fig. 5, el conjunto óseo del bloque temporal más antiguo es el más afectado por el óxido de manganeso, carbonato de calcio y el accionar de raíces, observándose una tendencia a la

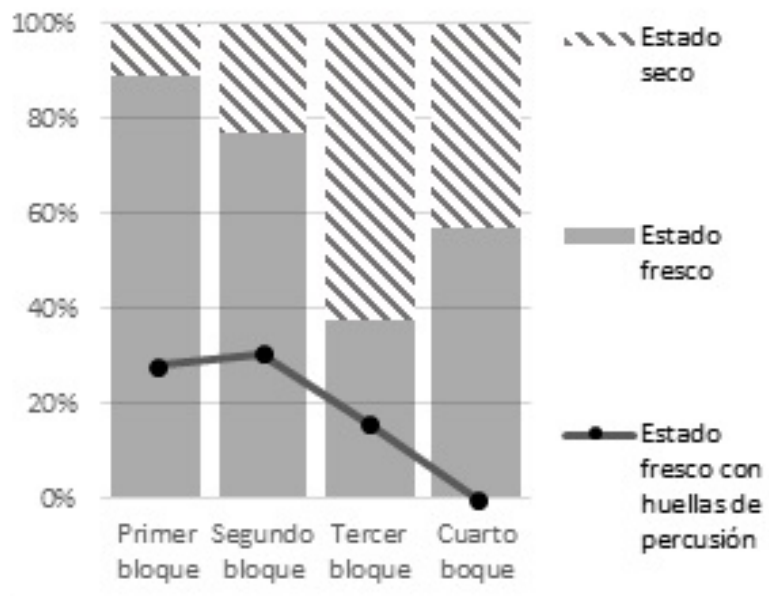

Fig. 7. Estado de fragmentación del NISP y huellas antrópicas asociadas.

disminución de estas modificaciones hacia los momentos más tardíos en el sitio, llegando a estar totalmente ausentes en el conjunto correspondiente al último bloque temporal aquí analizado. Para comprender cómo estas tres variables han actuado en la formación de los conjuntos es importante tener en cuenta la acción del agua en el sitio, debido a que todas estas modificaciones son producto y/o dependen, en cierta medida, de la existencia de condiciones de humedad (entre otros, López-González et al. 2006; Fernández-Jalvo \& Andrews, 2016). Debido a que RB1 se encuentra en un ambiente de características áridas y con un bajo régimen de precipitaciones, un leve aumento del ingreso de agua en el sitio podría resultar en un incremento de la vegetación, cambios en el pH del suelo y por lo tanto, en las condiciones que rodean a los restos influyendo, de este modo, en la velocidad del desarrollo de los procesos de diagénesis (Beisaw, 1998; Barrientos et al. 2007). Al respecto, la Dra. Montenegro (com. pers. en Guarido, 2014) indicó que la apertura de una grieta en el techo del alero habría permitido filtración de agua al sitio en algún momento de la secuencia, posiblemente con posterioridad a los entierros (Montenegro com. pers. en Guarido, 2014). Por otro lado, la Dra. Mehl y el Dr. Brook (com. pers. en Guarido, 2014) respaldan dicha propuesta, observando que la presencia de manganeso sobre los restos óseos de humanos sería resultado de la circulación de agua en el sitio y no de su estancamiento (Guarido, 2014; Mehl \& Franco, 2009). 
Como mencionamos en la sección sobre los antecedentes del sitio, hacia comienzos del primer bloque (entre ca. 4.200 y 4100 años AP), se registró en la estepa, en la zona de RB1, un incremento en la humedad producto del aumento en el régimen de precipitaciones (Franco et al. 2018) y, por tanto, condiciones más favorables para el crecimiento de plantas. Este periodo de humedad y de condiciones favorables finaliza hacia ca. 3.800 años AP -inicios del tercer bloque-, con el comienzo de un nuevo periodo caracterizado por condiciones áridas (Franco et al. 2017a, 2018). El hecho de que desde el primer hasta el tercer bloque temporal se haya registrado un alto porcentaje de radículas -a pesar de la considerable disminución de esta evidencia en el tercer periodosugiere que durante estos tres bloques temporales se habrían dado condiciones ambientales propicias para el crecimiento de plantas, es decir, estabilidad sedimentaria y condiciones lo suficientemente húmedas.

Al respecto, existe información acerca de que la descomposición de las raíces puede generar condiciones ácidas debido a la producción de ácido carbónico en el agua que circula entre los sedimentos (Tarbuck et al. 2005). Al aumentar la acidez, aumenta también la disolución de algunas sustancias con uniones químicas débiles, tales como el carbonato de calcio y el bicarbonato (Raiswell, 1987; Tarbuck et al. 2005). Cuando el agua circula por estratos con sedimentos con una alta presencia de carbonatos se forma una solución carbonatada, la cual, cuando el agua se evapora, precipita en forma de polvo y en algunos casos extremos forma concreciones cristalinas sobre los restos óseos (Hedges, 2002). Debido a que RB1 es un alero constituido en su totalidad por rocas sedimentarias con abundante contenido de material calcáreo (valvas de Ostreas), las cuales, a su vez, forman parte del sedimento (Mehl \& Franco, 2009), la matriz del sitio se vuelve susceptible a la disolución por la acidificación del sustrato, al menos en parte, producto de la putrefacción de las raíces (Guarido 2014). La disolución del material calcáreo en $\mathrm{RB} 1$ podría haber generado así, una saturación de iones de calcio y bicarbonato, los cuales habrían precipitado sobre los restos óseos de animales. Este proceso puede haber tenido lugar a lo largo de toda la secuencia de RB1 produciendo a su vez un efecto acumulativo en las capas inferiores, pertenecientes a los momentos más tempranos de la secuencia analizada. Asimismo, si las condiciones del sustrato varían (i.e. acidificación o alcalinización), los restos óseos pueden verse más o menos afectados por la precipitación de carbonato de calcio.

La notable disminución de marcas de raíces en la superficie de los huesos registrados a partir del segundo bloque temporal (entre ca. 4.100 y 3.900 años AP), podría relacionarse con la depositación de las cenizas del volcán Aguilera, que habría afectado negativamente el crecimiento de plantas. La menor representación de marcas de raíces sobre los especímenes óseos registrada hacia el tercer bloque temporal (ca. 3.800 a 3.600 años $\mathrm{AP})$, también podría ser resultado de este mismo proceso, sin embargo, el registro de condiciones de menor humedad y el comienzo de condiciones más áridas hacia ca. 3.800 años AP (Franco et al. 2018) habría acentuado, aún más, las condiciones desfavorables para el desarrollo de la vegetación en el sitio implicando así una mayor reducción de la cubierta vegetal.

Observamos que a medida que disminuye el accionar de raíces también lo hace la frecuencia de carbonato de calcio depositado sobre los especímenes óseos, lo cual estaría sugiriendo alguna dependencia entre estas variables. La disminución de ambas variables (Fig. 5) registrada a partir del segundo bloque (ca. 4.100 a 3.900 años $\mathrm{AP}$ ) podría estar relacionada tanto con la depositación de la ceniza del volcán Aguilera, como con el cambio de las condiciones ambientales hacia una mayor aridez (Franco et al. 2018). La acidificación de los suelos disminuiría debido a la menor presencia y descomposición de plantas y también a la menor circulación de solución carbonatada en los sedimentos (debido a un régimen de precipitaciones cada vez menor), lo que daría lugar a una menor depositación de carbonato de calcio sobre los restos óseos.

A su vez, se registró una elevada presencia de óxido de manganeso en los dos periodos más tempranos, una drástica disminución en el tercer bloque y su total ausencia en el cuarto bloque (Fig. 5). Nuevamente, a medida que disminuye el registro de óxido de manganeso se documenta una menor frecuencia de evidencia de marcas de raíces y de depósitos de carbonato de calcio. Si 
bien el óxido de manganeso puede ser producto de la descomposición de materia orgánica (Daniels, 1981; Fernández-Jalvo \& Andrews, 2016), también puede ser un subproducto del metabolismo microbiano (Daniels, 1981) y de la circulación de agua a través de las valvas de Ostreas del sedimento (Montenegro com. pers. en Guarido, 2014). Es decir, su registro implica condiciones propicias para la descomposición y actividad de bacterias, indicando la presencia de oxígeno, agua, sustratos con contenido orgánico y nutrientes inorgánicos; por lo tanto, su depositación sobre los restos óseos da cuenta de la existencia de condiciones reductoras en el microambiente que rodea a los huesos (Barrientos et al. 2007). En su tesis de licenciatura, Guarido (2014) relaciona la presencia de óxido de manganeso sobre los restos óseos de humanos con la disolución de valvas de Ostreas presentes en RB1, debido a que la filtración de agua proveniente de la grieta del techo habría contribuido a la precipitación de dicho óxido sobre los restos (Montenegro com. pers. en Guarido, 2014). Sin embargo, no descarta que la presencia de este último se deba al accionar de microorganismos vinculados a la putrefacción de la materia orgánica (Daniels, 1981, Myers \& Nelson, 1988 en Guarido, 2014). Esto mismo puede estar sucediendo también sobre los restos faunísticos, ya que la disolución de valvas de Ostreas habría tenido lugar a lo largo de toda la secuencia analizada, resultando en la precipitación tanto de óxido de manganeso como de carbonato de calcio. A su vez la actividad microbiana que acompaña el proceso de descomposición de las raíces antes mencionado, también podría haber contribuido produciendo una mayor presencia de óxido de manganeso entre los sedimentos con mayor presencia de plantas. En este sentido, la precipitación de óxido de manganeso habría dependido tanto del metabolismo microbiano como de la circulación de agua sobre los depósitos del alero.

El hecho de que el óxido de manganeso y el carbonato de calcio se presenten en porcentajes altos y varien a lo largo del tiempo, sugiere ciclos de condiciones alternantes de estabilidad, fluctuaciones en el ingreso de agua al sitio, cambios en el pH del sustrato $y$, en consecuencia, variaciones en la saturación de iones de bicarbonato de calcio (Hedges \& Millard, 1995; Pike et al.
2001; Hedges, 2002; Gifford-González, 2018). Estos ciclos afectarían no solo la depositación de sustancias, sino también los procesos de diagénesis de los huesos y su biodegradación (Hedges \& Millard, 1995; Pike et al. 2001; Hedges, 2002). Cabe mencionar que en ambientes muy saturados de calcio y fosfatos se ha registrado una tendencia menor a la desmineralización de la hidroxiapatita de los huesos (Hedges, 2002). Por tal motivo, si bien los restos óseos de RB1 pueden haber sufrido distintos procesos de biodegradación, el hecho de que el alero este compuesto de material calcáreo puede haber disminuido los efectos de la diagénesis en relación con la desmineralización de la hidroxiapatita de los huesos de animales depositados en el sitio.

En sintesis, la presencia de raíces y de agua circulando en el alero habrían producido condiciones lo suficientemente ácidas como para generar la depositación de carbonato de calcio y óxido de manganeso, los cuales registran valores más elevados en el primer bloque temporal (entre ca. 4.200 y 4.100 años AP), tienden a disminuir hacia el segundo y tercer periodo (entre ca. 4.100 a 3.600 años AP) y desaparecen por completo hacia el cuarto bloque (ca. 350 años AP). Si bien, esto sugiere que los conjuntos óseos más tempranos estuvieron sometidos a condiciones de mayor humedad, debe contemplarse también que la mayor frecuencia de carbonato de calcio y óxido de manganeso en el primer bloque puede deberse al efecto acumulativo de la depositación de sustancias, producto de la decantación de aquéllas transportadas por el agua desde las capas superiores hasta las inferiores.

Por otro lado, si bien a escala de sitio el carbonato de calcio podría haber actuado mitigando, al menos en parte, el deterioro de los huesos por la diagénesis, el hecho de que los porcentajes de especímenes afectados por dicha sustancia sean menores en el tercer bloque, en comparación con periodos previos, y que se encuentren completamente ausentes en el cuarto bloque, sugiere la posibilidad de que los dos conjuntos más tardíos tengan más chances de haber sufrido procesos diagenéticos relacionados con la pérdida de hidroxiapatita de los restos óseos, en comparación con los conjuntos más tempranos. Esto también podría relacionarse con el cambio 
en la forma del alero, dadas las inundaciones frecuentes a partir de ca. 542-328 cal. AP (Franco et al. 2018). De hecho, es probable que para el cuarto bloque temporal (ca. 350 años AP ó ca. 450 años cal. AP) la superficie sedimentaria del sitio y, tal vez, su protección, hayan sido menores, lo que haría que los restos depositados en este momento se encontraran más expuestos a las condiciones meteóricas. En el cuarto periodo no se registró evidencia del accionar de raíces, lo que puede relacionarse con la menor disponibilidad de humedad en estos momentos (Franco et al. 2018), sugiriendo que las condiciones ambientales podrían no haber sido adecuadas como para permitir el crecimiento de plantas, al menos en este sitio. Esto último concuerda con la evidencia de un avance glaciar menor hacia ca. 450 años cal. AP, el cual se reflejó en un aumento de la humedad en los Andes, acompañado de condiciones más áridas en la estepa (Aniya, 2013; Strelin et al. 2014 en Franco et al. 2018).

\section{Meteorización, evidencia de} carnivoros y roedores

La variación en los perfiles de meteorización de los bloques temporales sugiere la presencia de historias tafonómicas y posdepositacionales diferentes a lo largo de la secuencia de RB1. La comparación de los datos obtenidos indica la presencia de un alto porcentaje de especímenes de guanaco no meteorizados en los tres primeros bloques, sugiriendo un corto período de exposición a las condiciones meteóricas. En estos periodos predomina el estadio cero de meteorización; sin embargo, el hecho de que entre un $39 \%$ y un $24 \%$ de los especímenes de guanaco de dichos conjuntos alcanzara estadios más elevados indica cierto grado de exposición a las condiciones meteóricas, así como cierta destrucción de los restos óseos. Debido a que la frecuencia de elementos meteorizados no varía mucho dentro de los tres primeros bloques temporales, las pequeñas diferencias porcentuales podrían relacionarse tanto con la exposición de los conjuntos, como con su composición ósea interna, la cual puede derivar en grados variables de meteorización (Massigoge et al. 2010). Esta situación cambia en el cuarto bloque temporal, ya que la relación se invierte (Fig. 4).
Para dicho momento, el porcentaje de elementos meteorizados (incluyendo los estadios 1, 2, 3) se eleva considerablemente (67\%; Fig. 4). Cabe recordar que estos restos se encontraban ubicados muy superficialmente en la secuencia de RB1 y el hecho de que alli se hayan encontrado especímenes de Ovis dentro de un conjunto fechado en ca. 350 años AP (ó ca. 1.500-1.640 cal. DC), alerta sobre su potencial mezcla. Esto se debe a que, según los registros más tempranos disponibles sobre la introducción de la oveja en el área más cercana a RB1, corresponden a Punta Arenas (Chile) y esta se habría producido hacia 1.876-80 DC desde el estrecho de Magallanes, procedente de las islas Malvinas (Calderón Agez, 1936; Mateo Martinic, 2009), mientras que la fecha más temprana en la provincia de Santa Cruz (Argentina) es de 1.88589 DC y corresponde a Puerto San Julián (VV.AA. Centenario de Puerto San Julián, 2002).

En este sentido y tal como fue mencionado, las crecidas del río Bote registradas entre 542 y 328 años cal. AP pueden haber dado inicio a la erosión del sitio, provocando no solo la exposición de la estratigrafía, sino también la caída del techo, un cambio en la morfología del alero (Mehl \& Franco, 2009; Franco et al. 2018) y, en consecuencia, una variación en la tasa de depositación de los sedimentos eólicos debido a una mayor exposición a los vientos del oeste (Franco et al. 2016, 2018). De este modo, los restos depositados hacia el final de la secuencia de RB1 habrían estado sometidos a una dinámica mayor que el resto de los conjuntos, encontrándose así, más expuestos a las condiciones meteóricas. Estas condiciones pueden ser interpretadas como indicadoras de una baja estabilidad sedimentológica durante el cuarto bloque temporal, lo que explicaría no solo la menor evidencia de plantas, sino también la posibilidad de que este conjunto haya sufrido mayores alteraciones.

Por otra parte, en lo relativo al efecto de la composición etaria de los conjuntos óseos, cabe señalar que Massigoge et al. (2010) en sus estudios experimentales observaron que la meteorización puede afectar de manera diferencial a los distintos huesos del guanaco, ya que ésta está mediada por el desarrollo ontogénico del individuo. Según estos autores, los restos óseos de individuos crías y juveniles, por su estructura mineral y orgánica 
diferencial, son más propicios a alcanzar estadios de meteorización más avanzados en un período más corto de exposición a las condiciones meteóricas (Massigoge et al. 2010). Por tal motivo, si bien como sugiere Behrensmeyer (1978) la meteorización ósea es un indicador del grado de exposición relativo a las condiciones ambientales, la presencia de distintos estadios de meteorización no solo es el resultado de un aumento gradual en el tiempo de exposición y/o reexposición, sino que, como dicen Lyman \& Fox (1989), refleja la interacción de un conjunto de variables tafonómicas durante la formación de un conjunto óseo. En el caso de RB1, si bien no se registró una relación creciente entre el aumento de la meteorización y el incremento de especímenes sin fusionar en los tres primeros bloques temporales, el registro da cuenta de un elevado porcentaje de especímenes y de elementos cuya fusión no se completó. Por tal motivo, más allá de la ausencia de una relación clara entre meteorización y perfil etario, no descartamos la presencia de procesos tafonómicos vinculados con el desarrollo ontogénico del guanaco en los conjuntos óseos pertenecientes a los tres primeros periodos. Por otro lado, en el caso del bloque fechado en ca. 350 años AP, el incremento sustancial de la frecuencia de especímenes sin fusionar sugiere que el perfil de meteorización de los restos óseos de este conjunto, que es el más alto, es el que tiene más posibilidades de estar mediado por el perfil etario del guanaco.

En cuanto a la posibilidad de reexposición de los conjuntos, cabe mencionar que el accionar de roedores, así como el de carnívoros, es muy bajo en los tres primeros bloques y nulo en el cuarto periodo (Fig. 5). Lo anterior sugiere no solo que dichos agentes no serían los principales formadores de los conjuntos óseos, sino que tampoco habrían sido un factor perturbador de importancia, apoyando la idea de un enterramiento relativamente rápido de los restos y de una escasa o nula reexposición provocada por dichos agentes. Lo antes mencionado, en conjunto con la mayor frecuencia de huellas antrópicas en los tres primeros bloques (fig. 5), refuerza también la idea de conjuntos formados principalmente por la actividad humana. Cabe mencionar que la menor evidencia de carnivoros y roedores a partir del segundo bloque temporal, en comparación con el primer bloque, sugiere que dichos agentes no habrían frecuentado el sitio o lo habrían frecuentado en menor medida que durante el primer periodo. Esto puede llegar a ser un indicio acerca de que, a partir de este momento, habría una mayor continuidad en la ocupación humana de RB1, debido a que no se espera que los carnívoros hayan habitado RB1 junto con los humanos, lo que es concordante con la evidencia faunística proveniente de Chorrillo Malo 2 (Otaola, 2008, 2009) y con la ocupación efectiva de estos espacios, fechada entre 4.300 y 3.800 años AP, sostenida a partir de la evidencia lítica (Franco, 2002, 2004a y b; Franco et al. 2011).

\section{Fragmentación ósea}

No se encontró relación entre la fragmentación ósea -tanto de los conjuntos completos como en la de los especímenes de guanaco (Fig. 6 y 7)- y la meteorización de este último taxón a lo largo de la secuencia de RB1 (Gráfico 3). Incluso, el tercer bloque temporal (entre ca. 3.800 y 3.600 años AP), que es el que presenta el mayor NID y el mayor porcentaje de especímenes fracturados en estado seco del sitio, posee un alto porcentaje de elementos no meteorizados; en cambio el cuarto bloque temporal (ca. 350 años AP), el cual presenta el menor NID y un porcentaje mayor de especímenes fracturados en estado fresco, es el bloque más afectado por la meteorización. En este sentido, la mayor fragmentación ósea no parece estar produciendo conjuntos más meteorizados, como tampoco la meteorización parece estar incidiendo en una mayor fragmentación.

Sin embargo, es llamativo el elevado aumento del NSP y de la relación NSP/NISP en el tercer bloque temporal, donde se da un aumento en el porcentaje de especímenes fragmentados en estado seco y una disminución de la evidencia antrópica de fragmentación -huellas de percusión asociadas a especímenes fragmentados en estado fresco (Fig. 6 y 7)-. Esto sugiere la presencia de condiciones propicias para la fragmentación postdepositacional; tal como mencionamos, la presencia de improntas de raíces es mínima en este periodo, por lo cual la fragmentación del conjunto no parece ser producto de dicho agente. Al respecto, debe recordarse que el tercer bloque temporal es pennecontemporáneo 
a los entierros múltiples (fechados entre ca. 3.800 y 3.600 años AP) y por lo tanto, debe considerarse que el cavado de las estructuras de entierro implica la remoción de los sedimentos produciendo, entre otras cosas, la reexposición de los restos óseos a las condiciones meteóricas, la mezcla de materiales y la fragmentación posdepositacional de los huesos producto de su perturbación y movimiento. Si bien durante la excavación del sitio, los materiales contenidos en los pozos de entierro fueron separados del resto del conjunto que aquí se estudió, es probable que las actividades de sepultura sean las principales responsables de la mayor presencia de fracturas en estado seco del conjunto del tercer bloque, dando cuenta de una menor preservación respecto a los conjuntos previos. En este sentido, también es válido considerar que esa mayor fragmentación posdepositacional puede, además, ser la causa de una menor identificabilidad de indicadores de procesamiento antrópico sobre los especímenes. El estudio de los materiales contenidos en los pozos es parte de una próxima etapa de análisis sobre la que pensamos avanzar para una mejor comprensión de los procesos de cavado de los pozos y como ellos afectan el registro arqueológico previo.

\section{Densidad mineral ósea del guanaco}

Con respecto a la correlación \%MAU-DMO, los resultados obtenidos presentan diferentes valores a lo largo de la secuencia. El primer bloque temporal no registra una correlación estadísticamente significativa (ni para la porción axial o apendicular del esqueleto, ni para el total de los restos óseos en el conjunto). En cambio, durante el segundo y tercer bloque temporal, la correlación entre el \%MAU total de la muestra y la DMO de guanaco arrojó resultados positivos y estadísticamente significativos, alertando sobre la existencia de perfiles mediados por la densidad mineral ósea, caracterizados por una mayor frecuencia de restos de alta densidad. Sin embargo, al analizar cómo se comporta la DMO en el interior de estos conjuntos, se observa que la porción axial del esqueleto (porción que contiene los elementos de menor densidad ósea de toda la carcasa), no muestra una mayor representación de elementos de alta DMO. Por el contrario, la porción apendicular es la que registra una correlación alta, positiva y estadísticamente significativa, sugiriendo así, un mayor potencial de pérdida de elementos de menor densidad ósea vinculados a esta porción del esqueleto.

La situación antes descripta presenta un caso de equifinalidad, la cual en la literatura clásica (Binford, 1978; Lyman, 1984, 1992, 2004, 2008; Marean \& Frey, 1997; Wolverton, 2002; Marean \& Cleghorn, 2003; entre otros) ha sido vinculada principalmente a efectos de la DMO en conjunto con otros procesos tafonómicos, tales como el perfil etario del conjunto, el accionar de agentes como carnívoros y roedores y el procesamiento antrópico. Debe recordarse que, tanto la composición del sustrato del alero como el alto contenido de carbonato de calcio en los sedimentos, habrían favorecido la preservación de los restos óseos del primer, segundo y tercer bloque temporal, al mitigar el deterioro de los huesos por procesos diagenéticos que involucran la pérdida de hidroxiapatita.

Otra posible explicación podría involucrar la estructura etaria de los conjuntos de guanaco (Lyman, 1984, 1994b; Elkin \& Zanchetta, 1991; Álvarez et al. 2010; entre otros). Al respecto, diversos autores han propuesto que la DMO varía de manera considerable no solo entre especies, sino también entre los distintos elementos de un mismo individuo y entre los mismos elementos de un mismo taxón, en relación con el estado nutricional de cada individuo y el grado de osificación que cada elemento registra (Symmons, 2002; Ioannidou, 2003; Álvarez et al. 2010; Gutiérrez et al. 2009). De este modo, la mayor destrucción se produce sobre elementos con baja DMO (Lyman, 1994b). Sin embargo, como ya fue discutido, en nuestro caso no se registra un incremento considerable en el número de especímenes correspondientes a elementos sin fusionar, sugiriendo que, en los tres primeros bloques, los resultados de la relación \%MAU-DMO no estarían mediados por el perfil etario de los conjuntos.

Por otro lado, de manera general, los resultados de la correlación \%MAU-DMO pueden estar reflejando un perfil anatómico producto del accionar de agentes tales como carnívoros, roedores (Marean \& Cleghorn, 2003; Cleghorn \& Marean, 2004) e incluso el procesamiento humano intensivo 
de las carcasas (Outram, 2001, 2002; Wolverton, 2002). En el caso de RB1, como fue mencionado en párrafos previos, el accionar de carnívoros y roedores es muy bajo en los tres primeros bloques temporales e incluso nulo en el cuarto bloque, por lo que los resultados de la DMO no parecen estar mediados por el accionar de dichos agentes. En cuanto a la mediación de agentes antrópicos, si bien esta variable se interpreta con más detalle en el apartado siguiente, debe destacarse que en los tres primeros bloques se registró una elevada frecuencia de huellas antrópicas y, en el caso de los conjuntos comprendidos entre ca. 4.100 y 3.600 años AP -segundo y tercer periodo-, una elevada fragmentación de huesos en estado fresco con huellas de percusión asociadas (Fig. 6 y 7). Esto último sugiere que los resultados de la relación \%MAU-DMO del segundo y el tercer bloque pueden estar reflejando variaciones en el procesamiento antrópico del esqueleto apendicular, antes que los efectos negativos de la densidad mineral ósea -lo cual podría también estar explicando el considerable incremento del NID de estos momentos-.

Finalmente, como fue mencionado, no fue posible calcular la relación \%MAU-DMO para el cuarto bloque temporal, principalmente debido a la escasa representación de elementos óseos. Más allá de esta imposibilidad, los elementos que se encuentran representados son aquéllos con valores de densidad ósea media-elevada. Si consideramos esto en conjunto con los resultados de los análisis de las variables examinadas para este bloque temporal (condiciones ambientales y sedimentológicas menos estables, mayor deterioro por procesos diagenéticos y una mayor exposición a las condiciones meteóricas), el período fechado en ca. 350 años AP es el bloque con mayor destrucción y pérdida ósea.

\section{Evidencia antrópica}

En cuanto a la frecuencia y distribución de los diferentes tipos de huellas de procesamiento antrópico sobre elementos del guanaco, debe mencionarse que en el primer y segundo bloque temporal los resultados son muy similares. En ambos casos, la mayoría de las unidades anatómicas que se encuentran representadas poseen huellas de origen humano. Por el contrario, para el tercer bloque temporal, solo cuatro elementos del esqueleto axial poseen huellas antrópicas. Además, en este tercer periodo la distribución de huellas de corte en el esqueleto apendicular se ve restringida casi en su totalidad a las diáfisis. Finalmente, como ya se ha mencionado, a diferencia de momentos previos, en el cuarto bloque ningún taxón o espécimen indeterminado presenta evidencias de huellas antrópicas.

En los tres primeros bloques, las huellas de corte se registraron tanto en la porción axial como en la apendicular del guanaco, mientras que las de percusión se encuentran casi únicamente sobre la porción apendicular. Por otro lado, en los dos primeros bloques se registró una gran variabilidad en cuanto a la distribución de los diferentes tipos de huellas sobre el esqueleto apendicular. En el tercer bloque, a diferencia de lo que ocurre en periodos previos, la distribución de los diferentes tipos de huellas es poco variada; en este caso, la mayor frecuencia y tipos de marcas antrópicas se halla concentrada sobre las extremidades medias e inferiores.

La obtención de los diferentes recursos y nutrientes presentes en una carcasa debe ser entendida como un proceso que involucra varias etapas que pueden o no estar representadas en el mismo lugar (Binford, 1978; Mengoni Goñalons, 1999; Gifford-González, 2018, entre otros); debido a ello no se deben dejar de considerar las improntas óseas diferenciales y acumulativas de cada una de dichas etapas involucradas en el procesamiento de los conjuntos óseos. Al respecto, de acuerdo con el grado de desorganización al que se ven sometidos los elementos de guanaco y a la fragmentación registrada de los restos óseos, la evidencia sugiere que los conjuntos fechados entre ca. 4.200 y 3.900 años AP -primer y segundo bloque temporal-, corresponden al procesamiento final relacionado con la preparación y consumo de alimentos (Binford, 1978, 1981; Enloe, 1993; Mengoni Goñalons, 1999; De Nigris, 2004; Gifford-González 2018, entre otros). En cambio, durante el tercer bloque dicha relación no es tan clara debido a los diferentes procesos posdepositacionales identificados. Un factor que debe considerarse es que este tercer bloque temporal es pennecontemporáneo a los entierros de RB1 (Mehl \& Franco, 2009; Guarido, 2014; 
Franco et al. 2010, 2017a), por lo que la variación en los patrones registrados sobre el guanaco podría estar vinculado con algún factor idiosincrático o cultural (Binford, 1981).

En cuanto a la acción del fuego, la baja frecuencia de elementos termoalterados en todos los conjuntos de RB1 estaría sugiriendo la utilización de fuegos de baja temperatura para la cocción de huesos con carne. Además, los bajos porcentajes de elementos carbonizados y calcinados sugieren que la utilización de los restos óseos como combustible de fogones -como se ha señalado en tiempos etnohistóricos (Musters, 2005 [1911])- no sería importante.

\section{CONCLUSIONES}

En líneas generales, la evidencia arqueofaunística sugiere que durante el primer $y$ segundo bloque (entre ca. 4.200 y 3.900 años AP) se habrian dado condiciones más favorables para la preservación de los restos óseos. Esto se relaciona con una mitigación de los efectos de la diagénesis y la biodegradación de los restos óseos de animales debido tanto a la elevada presencia de carbonato de calcio circulando entre los sedimentos, como a la presencia de condiciones relativamente estables a nivel sedimentológico. A su vez, durante dicho lapso, los restos óseos de guanaco no se habrían visto afectados en gran medida por las condiciones meteóricas del ambiente debido a su rápido enterramientos, ni tampoco por la pérdida ósea producto de los efectos negativos de la densidad mineral diferencial de los huesos.

En cambio, a partir del tercer bloque (ca. 3.800 a 3.600 años AP) y de manera más notable en el cuarto bloque (ca. 350 años AP), las condiciones sedimentológicas y estratigráficas cambian. La menor estabilidad sedimentológica del tercer bloque, probablemente producto de la remoción de sedimentos para el entierro de los cuerpos humanos, dio como resultado un conjunto muy fragmentado por procesos posdepositacionales, lo que puede haber influido en la determinación anatómica y taxonómica, aunque no en la meteorización de los restos óseos, ni tampoco en los efectos de la densidad mineral ósea. Esto implica que se habría dado un mayor potencial de pérdida de restos óseos durante este período que en los dos bloques temporales previos. La preservación de los restos óseos por efectos diagenéticos aún no se vería comprometida debido a la alta presencia de carbonato de calcio en los sedimentos, hecho que, al igual que en los bloques previos, favorece la preservación de la hidroxiapatita de los restos óseos. Esta situación cambia hacia ca. 350 años AP, donde el registro no solo da cuenta del cambio en las condiciones sedimentológicas producto de una menor depositación de sedimentos, sino también en el ambiente de depositación de los restos óseos (ausencia de evidencia que sugiera un ambiente reductor, i.e.: carbonato de calcio y óxido de manganeso sobre los restos óseos). Para estos momentos es muy probable que debido a las inundaciones del río Bote, la morfología del alero haya cambiado y la columna sedimentaria haya estado más expuesta a las condiciones meteóricas. Además, una menor sedimentación acompañada de condiciones más áridas, habría provocado no solo un mayor potencial de mezcla y una mayor exposición a las condiciones meteóricas, sino también la ausencia de carbonato de calcio transportado por agua entre los sedimentos. Lo anterior puede haber desencadenado el desarrollo de procesos diagenéticos tales como la pérdida de hidroxiapatita de los restos óseos, lo que puede haber resultado en el perfil anatómico del guanaco mediado por los efectos negativos de la densidad mineral ósea observado para ca. 350 años AP.

Los carnívoros y roedores no habrían sido grandes perturbadores a lo largo de la secuencia de RB1. Por el contrario, la elevada frecuencia de huellas antrópicas en los tres primeros bloques temporales (ca. 4.200 y 3.600 años AP), sugiere que los humanos habrían sido el principal agente acumulador, al menos durante estos momentos. La evidencia de huellas antrópicas sugiere que durante los tres primeros periodos la carcasa del guanaco fue procesada, mientras que, en el cuarto bloque temporal, dicha presa no presenta señales claras de aprovechamiento humano.

En cuanto a las diferentes formas de ocupación de RB1, este trabajo apoya la idea de que RB1 habría sido usado al menos de dos formas diferentes por los grupos humanos que frecuentaban el área (Franco et al. 2017a, 2018). Es decir, en un primer momento -entre ca. 4.200 y 3.900 años AP (primer y segundo bloque 
temporal)- el registro faunístico del sitio sugiere que se habrían llevado a cabo actividades relacionadas con la subsistencia. Posteriormente a la erupción del volcán Aguilera (ca. 4.000 y 3.900 años AP), RB1 cambia su funcionalidad, usándose repetidamente como lugar de entierro de cuerpos humanos, luego de ca. 3.800 y 3.600 años AP (tercer bloque). Para estos momentos, las arqueofaunas del sitio sugieren el procesamiento humano del guanaco. En cuanto al cuarto bloque temporal, la presencia de un conjunto zooarqueológico poco preservado y con indicadores de mezcla no permite realizar interpretaciones confiables sobre el aprovechamiento faunístico para este momento. No obstante, la variación registrada con respecto a momentos previos refuerza la idea de un cambio en la forma de utilización del área, lo cual en conjunto con el registro de la presencia humana en sitios a cielo abierto en las cercanías del lago argentino sugiere la presencia de nuevos grupos humanos (Franco et al. 2017a, 2018), siendo los datos genéticos la evidencia más fuerte para asegurar que esto último habría sucedido (Franco et al. 2017b).

En resumen, el registro arqueofaunístico de RB1, a lo largo de la secuencia analizada, presenta variaciones en la preservación de los conjuntos zooarqueológicos. Como se puede observar, dichas variaciones se dan a la par de los cambios ambientales registrados en el área (Mancini, 2002; Ohlendorf et al. 2014; Franco et al. 2016, 2018).

Con respecto a esto último, es importante destacar que, los conjuntos zooarqueológicos de RB1 y de ChM2 tienen en común, principalmente, el registro del procesamiento antrópico del guanaco. De hecho, debe mencionarse que durante el periodo analizado en toda Patagonia centrosur el guanaco es la principal presa, registrando variaciones en su forma de explotación a lo largo del tiempo (Mengoni Goñalons 1999; De Nigris 2004; Rindel 2009, 2013; Marchionni 2013, entre otros). A su vez, los resultados alcanzados en este trabajo indican variaciones en la frecuencia de huellas antrópicas de dicho ungulado entre los conjuntos aquí analizados, principalmente aquellas referidas a la intensificación de la fragmentación de los huesos largos de dicho animal, las cuales deberán ser evaluadas en profundidad en trabajos posteriores.
Los análisis aquí realizados permitieron identificar la importancia del agente humano en la generación y configuración de los conjuntos estudiados. De esta manera, el presente trabajo aporta información novedosa y relevante sobre los procesos y agentes involucrados en la formación del sitio RB1. Sin embargo, la información de detalle tafonómico que aquí se analiza no solo es relevante para comprender la historia formacional del sitio en cuestión, sino que además aporta información importante para su comparación con estudios tafonómicos previos como los realizados en ChM2, lo cual contribuye a comprender las dinámicas ambientales del sur del río Santa Cruz durante el Holoceno medio y tardío.

\section{AGRADECIMIENTOS}

Agradecemos a la National Geographic Society (grant 8397-08), a la Universidad de Buenos Aires (UBACyT W1/0404 y 20020130100664BA), al CONICET-NSF 2014 (Resolución 3080), y a la University of Georgia Research Foundation por su apoyo financiero durante la excavación del sitio. También agradecemos a la University of Arizona por la realización de los primeros fechados de Río Bote1. A su vez, estamos agradecidos por la ayuda recibida de Patrimonio Cultural de Santa Cruz, al dueño y al personal de la estancia de Río Bote, especialmente a Berni Roil, Gerardo Povazsan, y a todas las personas que participaron en los trabajos de campo. Finalmente, queremos agradecer a la Dra. Nora Franco, quien dirigió los proyectos antes mencionados y contribuyó con la lectura crítica de este manuscrito, y a los dos evaluadores anónimos quienes con sus importantes comentarios $y$ sugerencias contribuyeron a mejorar el presente trabajo.

\section{BIBLIOGRAFÍA}

Álvarez, M. C., González, M., Massigoge, A., Kaufmann, C., y Gutiérrez, M. (2010). La densidad mineral ósea y la variabilidad ontogénica en guanaco (Lama guanicoe). Implicancias para la construcción de marcos de referencia en Zooarqueología. Zooarqueología a principios del siglo XXI: Aportes teóricos, metodológicos y casos de estudio, 95-106. 
Barrientos, G., Goñi, R., Zangrando, A., Del Papa, M., Guraieb, S. G., Arregui, M. J., y Negro, C. (2007). Human taphonomy in southern Patagonia: a view from the Salitroso lake basin (Santa Cruz, Argentina). Taphonomy and Zooarchaelogy in Argentina. Ed. M. A. Gutiérrez, L. Miotti, G. Barrientos, G. Mengoni Goñalons y M. Salemme. BAR International Series 160:187-201.

Behrensmeyer, A. K. (1978). Taphonomic and ecology information from bone weathering. Paleobiology, 4, 150-162.

Beisaw, A. M. (1998). Differential preservation and recovery: taphonomy of bone preservation at the Thomas/ Luckey site, Ashland, NY (Master's thesis, State University of New York at Binghamton, Anthropology Department).

Binford, L. R. (1978). Nunamiut Ethnoarehaeology. Academic Press.

Binford, L. R. (1981). Bones: Ancient Men and Modern Myths. Academic Press.

Borrero (1994-95). Arqueología de la Patagonia. Palimpsesto, 4, 9-69.

Borrero, L. A., y Franco, N. V. (2000). Cuenca superior del río Santa Cruz: perspectivas temporales. Desde el país de los gigantes. Perspectivas arqueológicas en Patagonia, 2, 345-356.

Borrero, L. A., Martin, F. M., y Vargas, J. (2005). Tafonomía de la interacción entre pumas y guanacos en el Parque Nacional Torres del Paine, Chile. Magallania (Punta Arenas), 33(1), 95-114.

Cain, C. R. (2005). Using burned animal bone to look at Middle Stone Age occupation and behavior. Journal of Archaeological Science, 32(6), 873-884.

Calderón, J. (1936). Historia de la industria ganadera en el territorio de Magallanes. Boletín del Ministerio de Agricultura, (10).

Cleghorn, N., y Marean, C. W. (2004). Distinguishing selective transport and in situ attrition: a critical review of analytical approaches. Journal of Taphonomy, 2(1), 43-67

Daniels, V. (1981). Manganese-containing stains on excavated pottery sherds. Masca Journal, 1(8), 230-231

De Nigris, M. E. (2004). El consumo en grupos cazadores recolectores. Un ejemplo zooarqueológico de Patagonia meridional. (Tesis Doctoral) Publicaciones de la Sociedad Argentina de Antropología, Buenos Aires.

De Nigris, M. E., y Goñalons, G. M. (2004). El guanaco como fuente de carne y grasas en Patagonia. Contra viento y marea. Arqueología de Patagonia, 469-476. Buenos Aires: Instituto Nacional de Antropología y
Pensamiento Latinoamericano and Sociedad Argentina de Antropología.

Elkin, D. C. (1995). Volume density of South American camelid skeletal parts. International Journal of Osteoarchaeology, 5(1), 29-37.

Elkin, D., y Zanchetta, J. R. (1991). Densitometría ósea de camélidos. Aplicaciones arqueológicas. En Actas del X Congreso de Arqueología Argentina (Vol. 3, pp. 195204).

Enloe, J. G. (1993). Ethnoarchaeology of marrow cracking: implications for the recognition of prehistoric subsistence organization. From bones to behavior: ethnoarchaeological and experimental contributions to the interpretation of faunal remains, 82-100.

Fernandez-Jalvo, Y., y Andrews, P. (2016). Atlas of taphonomic identifications: 1001+ images of fossil and recent mammal bone modification. Springer.

Fiel, M. V. (2019). El sitio Río Bote 1: Nuevos datos sobre el aprovechamiento del guanaco (Lama guanicoe) en el Holoceno medio (Patagonia, Argentina). Arqueología de la Patagonia: el pasado en las arenas, pp: 517-517.

Franco, N. V. (2002). Estrategias de utilización de recursos líticos en la cuenca superior del río Santa Cruz. (Tesis doctoral inédita, Universidad de Buenos Aires).

Franco, N. (2004a). La organización tecnológica y el uso de escalas espaciales amplias. El caso del sur y oeste de Lago Argentino. Temas de arqueología, análisis lítico, 101-144.

Franco, N. (2004b). Instituto Nacional de Antropología y Pensamiento Latinoamericano, y Sociedad Argentina de Antropología. (2004). Rangos de acción, materias primas y núcleos preparados al sur de Lago Argentino. Contra Viento y Marea. Arqueología de la Patagonia, 105-116.

Franco, N. (2008). La estructura tecnológica regional y la comprensión de la movilidad humana: tendencias para la cuenca del río Santa Cruz. Arqueología del extremo sur del continente americano. Resultados de nuevos proyectos, 119-154.

Franco, N. (2009). Informe de trabajo de campo en el marco del proyecto $\mathrm{N}^{\circ}$ 8397-08: New information aboutthe peopling of the Upper Santa Cruz basin (southern Patagonia, Argentina) and of the role of rockshelters in the area: the site Río Bote 1. Presentado a National Geographic.

Franco, N. V., y Borrero, L. A. (2003). Chorrillo Malo 2: initial peopling of the upper Santa Cruz basin, Argentina. Where the south winds blow. Ancient evidence of Paleo South Americans, 149-152. 
Franco, N.V., Borrero, L.A., Belardi, J.B., Carballo Marina, F., Martin, F., Campan, P., Favier Dubois, C., Stadler, N., Hernández, M.I., Cepeda, H., Muñoz, S., Borella, F., Muñoz, F. y Cruz, I. (1999). Arqueología del Cordón Baguales y sistema lacustre al sur del Lago Argentino. Prehistoria, 3, 65-86

Franco, N. V., Borrero, L. A., Brook, G. A., y Mancini, M. V. (2018). Changes in the Technological Organization and Human Use of Space in Southern Patagonia (Argentina) During the Late Holocene. En Lithic Technological Organization and Paleoenvironmental Change (pp. 301-320). Springer.

Franco, N., Borrero, L., y Mancini, M. (2004). Environmental changes and hunter-gatherers in southern Patagonia: Lago Argentino and Cabo Vírgenes (Argentina). Before farming, 2004(3), 1-17.

Franco, N. V., Brook, G. A., Mancini, M. V., y Vetrisano, L. (2016). Changes in lithic technology and environment in southern continental Patagonia: the Chico and Santa Cruz River basins. Quaternary International, 422, 5765.

Franco, N. V., Brook, G. A., Guraieb, S. G., Mancini, M. V., Guarido, A. L., Mehl, A., y Montenegro, T. (2017 a). Reuse of burial sites during the Late Holocene: evidence from multiple human burials at the Río Bote 1 rockshelter, Upper Santa Cruz river basin (Southern Patagonia, Argentina). Latin American Antiquity, 28(4), 476-494.

Franco, N.V, Cirigliano, N. y Ambrústolo, P. (2011). Semejanzas en tecnologías, diseños y prácticas funerarias al sur de la cuenca superior del río Santa Cruz: algunos ejemplos correspondientes al Holoceno tardío. Bosques, montañas y cazadores: investigaciones arqueológicas en Patagonia Meridional (pp. 155-178).

Franco, N. V., Galimany, J., Moraga, M., y Borrero, L. A. (2017 b). La integración de información cultural y genética en el sur de la cuenca superior del río Santa Cruz: primeros resultados. Décimas Jornadas de Arqueología de la Patagonia, 15.

Franco, N. V., Guarido, A. L., Guráieb, S. G., Martucci, M., y Ocampo, M. (2010). Variabilidad en entierros humanos en la cuenca superior y media del río Santa Cruz (Patagonia, Argentina). En XVII Congreso Nacional de Arqueología (pp. 1901-06).

Franco, N. V., y Vetrisano, L (2014). Lithics and Early Human Occupations at the Southern End of the Deseado Massif (Patagonia, Argentina). People, Culture in Ice Age Americas: New Directions in Paleoamerican Archaeolog (pp. 13-33).
Gifford-Gonzalez, D. (1989). Ethnographic analogues for interpreting modified bones: some cases from East Africa. Bone modification, 179-246.

(2018). An Introduction to Zooarchaeology. Springer International. Cham, Switzerland.

Guarido, A. L. (2014). Aproximación a los procesos de formación de los entierros humanos fechados entre ca. 3800 y 3600 años AP en Río Bote 1 (Tesis de Licenciatura inédita. Facultad de Filosofía y Letras. Universidad de Buenos Aires).

Gutiérrez, M. A. (2004). Análisis tafonómicos en el área Interserrana (provincia de Buenos Aires). (Tesis Doctoral inédita, Universidad Nacional de La Plata).

Gutiérrez, M., Kaufmann, C., Massigoge, A., Alvarez, M. C. y González, M. (2009). A Comparative Study of Bone Mineralization in Guanaco (Lama guanicoe). Implications for Differential Preservation in Archaeological Sites. Facultad de Ciencias Sociales (UNCPBA), Olavarría. MS.

Grayson, D. K. (1984). Quantitative Zooarchaeology. Academic Press, New York

Hedges, R. E. M. (2002). Bone diagenesis: An overview of process. Archaeometry, 44, 319-328.

Hedges, R.E.M. y A.R. Millard (1995). Bones and groudwater: towards the modelling of diagenetic processes. Journal of Archaeological Science, 22, 155-164.

Ioannidou, E. (2003). Taphonomy of animal bones: species, sex, age and breed variability of sheep, cattle and pig bone density. Journal of Archaeological Science, 30(3), 355-365.

Johnson, E. (1985). Current developments in bone technology. En Advances in archaeological method and theory (pp. 157-235). Academic Press.

Johnson, E. (1989). Human modified bones from early southern Plains Sites. Bone modification, 431-471.

Kaufmann, C. A. (2009). Estructura de edad y sexo en guanaco: estudios actualísticos y arqueológicos en Pampa y Patagonia. Sociedad Argentina de Antropología.

López-González, F., Grandal-D`Anglade, A. y Vidal-Romani, J. R. (2006). Deciphering bone do positational sequences in caves trought the study of manganese coatings. Journal of Archaeological Science, 33, 707-717.

Lyman, R. L. (1984). Bone density and differential survivorship of fossil classes. Journal of anthropological Archaeology, 3(4), 259-299.

Lyman, R. L. (1992). Anatomical considerations of utility curves in zooarchaeology. Journal of Archaeological Science, 19(1), 7-22. 
Lyman, R. L. (1994a). Quantitative units and terminology in zooarchaeology. American Antiquity, 36-71.

Lyman, R. L. (1994b). Vertebrate taphonomy. Cambridge University Press.

Lyman, R. L. (2004). The concept of equifinality in taphonomy. Journal of Taphonomy, 2(1), 15-26.

Lyman, R. L. (2008). Quantitative paleozoology. Cambridge University Press.

Lyman, R. L., y Fox, G. L. (1989). A critical evaluation of bone weathering as an indication of bone assemblage formation. Journal of Archaeological Science, 16(3), 293-317.

Mancini, M. V. (2002). Vegetation and climate during the Holocene in Southwest Patagonia, Argentina. Review of Palaeobotany and Palynology, 122(3-4), 101-115.

Mancini, M. V., Franco, N. V., y Brook, G. A. (2013). Palaeoenvironment and early human occupation of southernmost South America (South Patagonia, Argentina). Quaternary International, 299, 13-22.

Marean, C. W., y Cleghorn, N. (2003). Large mammal skeletal element transport: applying foraging theory in a complex taphonomic system. Journal of taphonomy, 1(1), 15-42.

Marean, C. W., y Frey, C. J. (1997). Animal bones from caves to cities: reverse utility curves as methodological artifacts. American Antiquity, 698-711.

Arroyo, A. M., Ruiz, M. L., Bernabeu, G. V., Román, R. S., Morales, M. G., y Straus, L. G. (2008). Archaeological implications of human-derived manganese coatings: a study of blackened bones in El Mirón Cave, Cantabrian Spain. Journal of Archaeological Science, 35(3), 801-813.

Massigoge, A., González, M., Kaufmann, C. y Gutiérrez, M. (2010). Observaciones actualísticas sobre meteorización ósea en restos esqueletales de guanaco. Pasado y presente desde la arqueología pampeana, Tomo I, pp. 309-322.

Mateo Martinic, B. (2009). Documentos inéditos para la historia de Magallanes. Diario de vida de William Blain ovejero en Tierra del Fuego (1891-1898). Magallania, 37(1),199-221.

Mehl, A., y Franco, N. V. (2009). Cambios en la morfología de los reparos rocosos: el caso de los sitios arqueológicos Chorrillo Malo 2 y Río Bote 1 (pcia. de Santa Cruz, Argentina). Arqueología de Patagonia: Una mirada desde el último confín, 2, 893-900.

Goñalons, G. L. M. (1999). Cazadores de guanacos de la estepa patagónica. Sociedad Argentina de Antropología.

Goñalons, G. L. M. (2010). Zooarqueología en la práctica: algunos temas metodológicos. Xama, 19, 83-113.

Montalvo, C. L. (2002). Root traces in fossil bones from the Huayquerian (Late Miocene) faunal assemblage of Telén, La Pampa, Argentina. Acta Geológica Hispánica, 37-42.

Morlan, R. E. (1980). Taphonomy and archaeology in the upper Pleistocene of the northern Yukon Territory: a glimpse of the peopling of the New World. National Museum of Man Archaeological Survey of Canada.

Musters, G. C. (2005 [1911]). Vida entre los Patagones. El Elefante Blanco.

Ohlendorf, C., Fey, M., Massaferro, J., Haberzettl, T., Laprida, C., Lücke, A., Maidana, N., Mayr, C., Oehlerich, M., Ramón Mercau, J., Wille, M., Corbella, H., St-Onge, G., Schäbitz, F. y Zolitschka, B. (2014). Late Holocene hydrology inferred from lacustrine sediments of Laguna Cháltel (southeastern Argentina). Palaeogeography, Palaeoclimatology, Palaeoecology, 411, 229-248.

Otaola, C. (2008). Explotación faunística durante el Holoceno medio y tardio en el sitio Chorrillo Malo 2, Provincia de Santa Cruz, Argentina. ¿Variaciones o continuidades?. (Tesis de licenciatura inedita. Universidad de Buenos Aires).

Otaola, C. (2009). Sitio Chorrillo Malo 2, Provincia de Santa Cruz, Argentina: Enfoque arqueofaunístico de un sitio en un" callejón sin salida". Arqueología de Patagonia: una mirada desde el último confín, 355-365.

Otaola, C., y Franco, N. V. (2008). Procesamiento y consumo de guanaco en el sitio Chorrillo Malo 2, provincia de Santa Cruz, Argentina. Magallania, 36(2), 205-219.

Outram, A. K. (2001). A new approach to identifying bone marrow and grease exploitation: why the "indeterminate" fragments should not be ignored. Journal of archaeological science, 28(4), 401-410.

Outram, A. K. (2002). Bone fracture and within-bone nutrients: an experimentally based method for investigating levels of marrow extraction. Consuming passions and patterns of consumption, (pp. 51-64). McDonald Institute for Archaeological Research.

Pike, A., C. M. Nielsen-Marsh y R.E.M. Hedges 2001. Modelling bone dissolution under different hydrological regimes. Archaeological Sciences '97. Proceedings of the conference held at the University of Durham $2^{\text {nd }}$ - $4^{\text {th }}$ September 1997. British Archaeological Reports, International Series 939. Archaeopress.

Raiswell, R. (1987). Non-steady state microbiological diagenesis and the origin of concretions and nodular limestones. Geological Society, London, Special Publications, 36(1), 41-54 
Shipman, P. (1981). Applications of scanning electron microscopy to taphonomic problems. Annals of the New York Academy of Sciences, 376(1), 357-385.

Stine, S. (1994). Extreme and persistent drought in California and Patagonia during Medieval Time. Nature, 369, 546-549.

Strelin, J. A., Kaplan, M. R., Vandergoes, M. J., Denton, G. H., y Schaefer, J. M. (2014). Holocene glacier history of the Lago Argentino basin, southern Patagonian Icefield. Quaternary Science Reviews, 101, 124-145.

Symmons, R. (2002). Bone Density Variation between Similar Animals and Density Variation in Early Life: Implications for Future Taphonomic Analysis. Biosphere to lithosphere: New studies in vertebrate taphonomy, (pp. 86-93).

Tarbuck, E.J., Lutgens, F.K. y Tasa, D. (2005). Ciencias de la Tierra. Pearson Educación S.A., Madrid.

Vetrisano, L. (2017). La producción laminar en la cuenca media y superior del río Santa Cruz (Patagonia). (Tesis de Licenciatura inédita, Universidad de Buenos Aires).

VV.AA. (2002).Centenario de Puerto San Julián. 1901 2001. Una ventana al pasado. Provincia de Santa Cruz. Patagonia argentina. Tomo I.

Wolverton, S. (2002). NISP:MNE and\% whole in analysis of prehistoric carcass exploitation. North American Archaeologist, 23(2), 85-100. 